\title{
DECISION MAKING PROCESS FOR SERVING RESTAURANTS USING INTUITIONISTIC FUZZY SET THEORY VIA CONTROLLED SETS
}

\author{
Gökhan ÇUVALCIOĞLU ${ }^{1}$ and Serkan Ural VAROL ${ }^{2}$ \\ 0000-0001-5451-3336 and 0000-0003-4944-1440
}

\begin{abstract}
The Intuitionistic fuzzy set theory gives quite successful results in decision-making processes when compared to other set theories. For this reason, it finds application areas in many areas of daily life such as political science, robotic systems, economic research, medical studies. The success of the IFS concept in decision-making processes in these areas has also been proven. In this study, it is aimed to create an IFS model that can make suggestions to support the end user in the process of choosing a product. Hamming measure will be used to achieve this goal. As per the definition of this measure, the degree of non-membership of the data is as important as the degree of membership. These values also determine the degree of intuition. However, the easier it is to estimate the yield value of a property of an object, the more difficult it is to estimate the non-fulfillment value. For this reason, in this study, the data will be intuitiveized by the controlled set theory and the relationships with the results will be determined. In all these processes The Microsoft SQL Server data structure for coding was used and algorithms were created according to this coding. A healthy evaluation of the data is as important as the value of the data's feature of not having that feature. This situation is the most important factor determining the value of intuition.
\end{abstract}

\section{INTRODUCTION}

The concept of fuzzy cluster, membership degrees and the degree of non-membership given by the fuzzy logic rules are easily obtained. Thus, the classification of an object is easily made over a chain, specifically unit range. However, the heuristic fuzzy set theory revealed that this situation would not be so clear and the degree of intuition was also important. The biggest problem in this theory is determining the degree of non-membership rather than determining the degree to which an object has a feature. Because the degree of intuition also appears depending on these two values. In fact, in heuristic fuzzy set theory, the fact that an object has a property is explained by two independent variables and the third dependent variable connected to them. The biggest challenge faced by many scientists working in this field is choosing two independent variables separately. Because this choice determines the degree of intuition. For example, in determining the height degree of a person whose height is $180 \mathrm{~cm}$ as 0.825 and the degree of not being as 0.175 , what criterion has been set for the value 0.175 ? The intuition level for this person is 0.1 .

Date: Received: 2021-07-28 Accepted: 2021-08-01

2000 Mathematics Subject Classification. 03E72

Key words and phrases: Decision making, Controlled set, Intuitionistic fuzzy set, Hamming distan 
Is this intuition a human intuition? It is clear that the answer is no. Because, some different methods can be followed in determining the degree of membership, but the degree of non-membership should be linked to our intuition. It is our experience that strengthens our intuition. Then the degree of nonmembership of a person whose height is $180 \mathrm{~cm}$ should vary according to the group to which he belongs. For example, a value of 0.175 may be acceptable according to people in a city, but in the group with basketball athletes this value is 0 or the membership level should be re-examined.

To solve the above-mentioned situation, controlled sets were defined by Çuvalcıoğlu [3] in 2014, and the fact that an object has a property is expressed by one independent two dependent variables.

Due to the conflicts in the results obtained in the decision-making processes made so far, some improvements have been made or given as they are. The reason for this is that the degree of nonmembership and the degree of intuition cannot be determined exactly. However, distance measures use all three values. In that case, the relationship results to be obtained from the distance measures by not being a member closest to the line and using the degree of intuition will be successful. The resulting models will have a more similar structure to human intuition. For distance measurements, see $[4,5]$ respectively.

In this study, the purpose of using this method is to successfully complete the relation of hundreds of features of thousands of products with hundreds of products in a short time and successfully.

The article is organized as follows. In Chapter 2, some basic concepts are given, and in Chapter 3, the controlled set theory to be used in heurization is mentioned. In addition, information was given about the dimensions and properties used in the study. In the section, coding, obtaining the results and evaluating the results were made.

\section{PRELIMINARY PREPARATIONS}

We recall some basic concepts of IFS, [1, 2].

Definition 2.1. A fuzzy set $A$ in a nonempty set $X$ is an object having the form $A=\left\{\left(x, \mu_{A}(x), 1-\right.\right.$ $\left.\left.\mu_{\mathrm{A}}(\mathrm{x})\right) \mathrm{xX}\right\}$

where the function $\mu_{\mathrm{A}}: \mathrm{X} \rightarrow[0,1]$ denoted the degree of membership and $1-\mu_{\mathrm{A}}$ denoted degree of non membership degree of $\mathrm{x}$.

Definition 2.2. An Intuitionistic fuzzy set ( briefly I F S see[1]) A in a nonempty set $X$ is an object having the form $A=\left\{\left(x, \mu_{A}(x), \gamma_{A}(x)\right) x X\right\}$ where the functions $\mu_{A}, \gamma_{A}: X \rightarrow[0,1]$ denote the degree of membership and degree of non membership, respectively and $0 \leq \mu_{A}(x), \gamma_{A}(x) \leq 1$ for all $x \in X$. The value $\pi_{A}(x)=1-\mu_{A}(x)-\gamma_{A}(x)$ is called hesitation degree of $x$.

Example 2.3. Let $\mathrm{X}=\{\mathrm{a}, \mathrm{b}, \mathrm{c}, \mathrm{d}\}$ an universal and

$$
A=\{(a, 0.875,0.125),(b, 0.54,0.16),(c, 0.25,0.35),(d, 0.95,0.0)\}
$$

Intuitionistic fuzzy set on X.The hesitation degrees of $\mathrm{a}, \mathrm{b}, \mathrm{c}, \mathrm{d} \in \mathrm{X}$ are as follow, respectively

$$
\pi_{A}(a)=0, \pi_{A}(b)=0.3, \pi_{A}(c)=0.4, \quad \pi_{A}(d)=0.05
$$

Definition 2.4. Let $A, B \in$ IFS (X).
i. $A \subseteq B \Leftrightarrow \mu_{A}(x) \leq \mu_{B}(x)$ ve $\gamma_{A}(x) \gamma_{B}(x) \forall x \in X$.
ii. $A=B \Leftrightarrow A \subseteq B$ ve $B \subseteq A$
iii. $A^{c}=\left\{\left(x, \gamma_{A}(x), \mu_{A}(x)\right) x X\right\}$
iv. $A B=\left\{\left(x, \max \left\{\mu_{A}(x), \mu_{B}(x)\right\}, \min \left\{\gamma_{A}(x), \gamma_{B}(x)\right\}\right) \mid x \in X\right\}$ 
v. $A B=\left\{\left(x, \min \left\{\mu_{A}(x), \mu_{B}(x)\right\}, \max \left\{\gamma_{A}(x), \gamma_{B}(x)\right\}\right) \mid x \in X\right\}$

Örnek 2.5. $X=\{a, b, c\}$ be an universal.

$\mathrm{A}=\{(\mathrm{a}, 0.45,0.25),(\mathrm{b}, 0.75,0.25),(\mathrm{c}, 0.50,0.15)\}$,

$B=\{(a, 0.35,0.15),(b, 0.90,0.05),(c, 0.65,0.45)\}$

Intuitionistic fuzzy sets on $\mathrm{X}$. Then,

$\mathrm{A}^{\mathrm{c}}=\{(\mathrm{a}, 0.25,0.45),(\mathrm{b}, 0.25,0.75),(\mathrm{c}, 0.15,0.50)\}$

$\mathrm{AB}=\{(\mathrm{a}, 0.45,0.15),(\mathrm{b}, 0.90,0.05),(\mathrm{c}, 0.65,0.15)\}$

$\mathrm{AB}=\{(\mathrm{a}, 0.35,0.25),(\mathrm{b}, 0.75,0.25),(\mathrm{c}, 0.50,0.45)\}$

\section{CONTROLLED SET THEORY}

Hesitation value is of great importance in applications made using more data than theoretical studies on intuitionistic fuzzy sets, in the general name of decision-making processes. As can be seen from its definition, the calculation of the hesitation value is directly related to the membership degree and non-membership degree. In conventional methods, whether the degree of hesitation is effective on the degree of membership or non-membership is not taken into account. In many studies, the degree of non-membership is tried to be given without any justification. That is, the more concrete the determination of the degree of membership, the more abstract the degree of non-membership or, provided that the condition is met, it is random. This situation negatively affects the role of the hesitation value of the data in the decision-making process.

The Hamming measure to be used in this study works on all these values. Therefore, not only membership degree but also non-membership and hesitation value will be decisive for interpreting the decision-making process in a way that is close to correct.

Due to the situations discussed above, the method of forming controlled sets will be used to consistently determine the non-membership and hesitation value using the membership degrees of the obtained data. Controlled sets were first described by Çuvalcıŏlu [3] in 2014.

Zadeh's example about long peoples, the membership degree of one whose lenght $170 \mathrm{~cm}$ is almost 1, say 0.8. But, if we choose the universal as the people whose lenght is longer than $170 \mathrm{~cm}$ then the membership degree of the person $171 \mathrm{~cm}$ tall is subject of discussion. Because, in this universal, while the membership degree of the person $190 \mathrm{~cm}$ tall is almost 1 , how can we say taht the membership degree of the person $171 \mathrm{~cm}$ tall is 0.8 ? In Zadeh's example $\mu(171)=0.8,1-\mu(171)=0.2$. But, if we choose the universal as above, then we are in the expectations that the universal have an element which membership degree is 0.2 . Hence, the membership degree of the persons which $171 \mathrm{~cm}$ is 0.8 . This is a contradiction. In this statement, the best solution can be the membership degree of the persons which $171 \mathrm{~cm}$ is 0.5 . Also in this case, the membership degree of the persons which $180 \mathrm{~cm}$ is 0.9 thus the non-membership degree of the persons which $180 \mathrm{~cm}$ is 0.1 . This is a contradiction, too.

This problem can be solve by defining a bijective and order preserving function between the image of fuzzy set on Zadeh's universal as above and the image of fuzzy set on subset of Zadeh's same universal. However, in this case, problems associated with the concept appears to be taller. So, in order to find any element in universal with its membership and non-membersip degree, there must also have an element such that it control the other. With this idea, if we use the Zadeh's universal for the long people then the membership degree of the persons which $171 \mathrm{~cm}$ is 0.8 and thus the nonmembership degree of the persons which $171 \mathrm{~cm}$ is 0.2 . But, If we use the subuniversal of the Zadeh's universal then the membership degree of the persons which $171 \mathrm{~cm}$ is 0.8 and thus the non- 
membership degree of the persons which $171 \mathrm{~cm}$ is 0.0 . Because, there is not an element which control the non-membership degree of the persons which $171 \mathrm{~cm}$.

An extension of the fuzzy theory is the intuitionistic fuzzy theory which have the hessitation degree that is not belong the fuzzy theory. But, there are same problems in Intuitionistic fuzzy theory like Fuzzy theory's. Because, there is not any criterion for non-membership degree of an element. For example, the set $A=\{(x, 0.8,0.2),(y, 0.4,0.3)\}$ is an intuitionistic fuzzy set on $U=\{x, y\}$. But, there is not any controller element for $\mathrm{x}$, like $\mathrm{y}$.

As a result of these discussions, controlled sets were defined by Çuvalcığlu [3] as follows.

Definition 4.1: Let $E$ be an universal, $\mu$ is a fuzzy set on $E$. The set $E$ is called $\mu$-controlled set if $\forall \mathrm{x} \in \mathrm{E}, \exists \mathrm{y} \in \mathrm{E} \ni 1-\mu(\mathrm{x})=\mu(\mathrm{y})$.

If $E$ is $\mu$-controlled set then we write $\operatorname{E} \in \operatorname{CS}(\mu)$.

Example4.2: Let $\mathrm{X}=\{\mathrm{a}, \mathrm{b}, \mathrm{c}, \mathrm{d}, \mathrm{e}, \mathrm{f}\}$ be an universal.

$A=\{(a, 0.45),(b, 0.05),(c, 0.50),(d, 0.35),(e, 0.90),(f, 0.65)\}$ is not a controlled set. But,

$B=\{(a, 0.45),(b, 0.10),(c, 0.50),(d, 0.45),(e, 0.90),(f, 0.55)\}$ is a controlled set. In $B, a$ is controled by $\mathrm{f}, \mathrm{c}$ is controlled by itself, $\mathrm{f}$ controlled by a and $\mathrm{d}$, etc.

\subsection{Controlled sets on classical set theory}

In this study, it has been shown that the $\mathcal{C}=\{\mathrm{X} \subset \mathrm{E}: \mathrm{X} \in \mathrm{CS}(\mu)\}$ family has the maximal element property. We define the set $\tilde{a}=\{b \in E: 1-\mu(a)=\mu(b)\}$.

Proposition4.1.1: Let $\mathrm{E}$ be an universal, $\mu$ is fuzzy set, $\mathcal{C}=\{\mathrm{X} \subset \mathrm{E}: \mathrm{X} \in \operatorname{CS}(\mu)\}$ and $\mathrm{A} \in \mathcal{C}$. For $\mathrm{a} \in \mathrm{A}$, we define $\mathcal{C}^{\prime}=\left\{\mathrm{Y}_{\mathrm{a}}: \mathrm{a} \in \mathrm{A}\right\} \subset \mathcal{C}$ where ă $=\{c a ̃ ~: c A\}, Y_{\mathrm{a}}=\{\mathrm{a}\} \cup a ̆$ then, we get $\mathrm{A}=\cup \mathcal{C}^{\prime}$

With the above proposition, it can be easly seen the family $\mathcal{C}$ has a base as following,

where $\widetilde{A}=U_{a \in A}$ a .

$$
\underline{A}=A \cup \widetilde{A}
$$

Theorem 4.1.2: Let $E$ be an universal, $\mu$ is fuzzy set, $A \in \mathcal{P}(E)$. The mapping J: $P(E) \rightarrow P(E)$ defined by $\mathrm{J}(\mathrm{X})=\underline{\mathrm{X}}$ is a closure operator.

From this theorem, it is concluded that the $\mathcal{C}$ family is a closed system, $(\mathcal{C}, \subseteq)$ is complete lattice, and every closed subsystems $C^{\prime}$ of $\mathcal{C}$ is complete lattice. As a result of these, it was obtained that $\mathcal{C}$ is a Moore family. It has also been shown that the $\mathcal{C}$ closed system is algebraic. According to Schimid's theorem $\mathcal{C}$ closed system is inductive, as a result every chain in $\mathcal{C}$ have a supremum in $\mathcal{C}$.

As a result of these properties, it can be easily seen that cluster theoretic properties are workable on controlled sets.

\section{2. $\left(\alpha, \alpha^{*}\right)$-Controlled sets}

Another problem is whether a fuzzy set can be created as a controlled set. Considering the studies on intuitionistic fuzzy sets, the main reason for this problem can be explained as follows, 
The membership degree is very important for an element in any set. But the non-membership degree is very important, too. We can not claim that all sets are controlled set. However, it is possible to construct a set in such a way that it can have a controlled set property. We can introduce controlled set using the membership degrees of elements. The study on the solution of this problem is given by Çuvalcıoğlu [3] as follows.

Definition 4.2.1: Let $\mathrm{E}$ be an $\alpha$-set. We define the following mapping on $\mathrm{E}$ as following,

$$
\alpha^{*}(\mathrm{x})=\left\{\sup _{\mathrm{y} \in \mathrm{E}} \alpha(\mathrm{y}), \alpha(\mathrm{x}) \leq 1-\alpha(\mathrm{y}) 0 \quad\right. \text {, otherwise }
$$

It is clear that $\alpha^{*}$ is a mapping from $E$ to I. In addition, it can be easily seen that the sum of $\alpha$ and $\alpha^{*}$ is less or equal than 1. From this properties, we can give the following definition

Definition 4.2.2: Let $\mathrm{E}$ be $\alpha$-set. Then the set $\mathrm{A}=\left\{<\mathrm{x}, \alpha(\mathrm{x}), \alpha^{*}(\mathrm{x}) \mathrm{x} \in \mathrm{E}\right\}$ is called $\left(\alpha, \alpha^{*}\right)$-controlled set.

Example 4.2.3: If we use the set $A$ used in above example, $A$ is not a controlled set. But if we use the definition5. We get a new set $A^{*}$ of which element's membership degrees have the same membership with the same elements of A

$\mathrm{A}^{*}=\{(\mathrm{a}, 0.45,0.50),(\mathrm{b}, 0.05,0.90),(\mathrm{c}, 0.50,0.50),(\mathrm{d}, 0.35,0.65),(\mathrm{e}, 0.90,0.05),(\mathrm{f}, 0.65,0.35)\}$

In this set, $\mathrm{a}$ and $\mathrm{c}$ are controlled by $\mathrm{c}$, e is controlled by $\mathrm{b}$, etc.

If we examine the controlled set $B$ using Definition 5., then we get, $B^{*}=$ $\{(\mathrm{a}, 0.45,0.55),(\mathrm{b}, 0.10,0.9),(\mathrm{c}, 0.50,0.50),(\mathrm{d}, 0.45,0.55),(\mathrm{e}, 0.90,0.10),(\mathrm{f}, 0.55,0.45)\}$ is a controlled set. In $\mathrm{B}^{*}$, like as $\mathrm{B}$, a is controled by $\mathrm{f}, \mathrm{c}$ is controlled by itself, $\mathrm{f}$ controlled by a and $\mathrm{d}$, etc.

From the definition, it can be easily seen that every $\left(\alpha, \alpha^{*}\right)$-controlled set is an intuitionistic fuzzy set. But the converse of this is not true generaly.

\section{HAMMING DISTANCE BETWEEN IFSS}

In this section, we will provide information about the Hamming measure between IFSs, which we will use in the process of deciding the relationship between our data. The hamming measure, like other measures, can be associated with a similarity measure between IFSs..

Tanım 5.1: Let $\mathrm{X}$ be a nonempty set and A, B, C IFS (X). The distance measure between A and $\mathrm{B}$ is a function d: IFS $\times$ IFS $\rightarrow[0,1]$.

i. $0 \mathrm{~d}(\mathrm{~A}, \mathrm{~B}) 1$

ii. $d(A, B)=0 A=B$

iii. $d(A, B)=d(B, A)$

iv. $d(A, C)+d(B, C) \geq d(A, B)$

v. If $A \subseteq B \subseteq C$ then $d(A, C) \geq d(A, B)$ and $d(A, C) \geq d(B, C)$.

Hamming measure is defined as follow;

$\mathrm{d}(\mathrm{A}, \mathrm{B})=\sum_{\mathrm{i}=1}^{\mathrm{n}} \quad\left(\left|\mu_{\mathrm{A}}(\mathrm{x})-\mu_{\mathrm{B}}(\mathrm{x})\right|+\left|\gamma_{\mathrm{A}}(\mathrm{x})-\gamma_{\mathrm{B}}(\mathrm{x})\right|+\left|\pi_{\mathrm{A}}(\mathrm{x})-\pi_{\mathrm{B}}(\mathrm{x})\right|\right)$

6. MAIN RESULTS

In this study, the Hamming measure will be run on controlled sets. Therefore, in this section, first of all, controlled sets will be created with the data obtained by experts in the field. For this purpose, blurring will be done with SQL (Structured Query Language) using Microsoft SQL Server program.

\subsection{Case study.}




\section{DECISION MAKING PROCESS VIA CONTROLLED SETS}

Let $A=\{G R 1, G R 2, G R 3, G R 4, G R 5\}$ grape varieties,

Let $C=\{\mathrm{x} \mid \mathrm{x}$ property of beverage product $\}$ be properties of product obtained from grapes.

Let $S=\{x \mid x$ types of food $\}$ be the set of foods to be consumed according to product characteristics.

The membership degrees of the relations of these data with each other were determined by experts as follows.

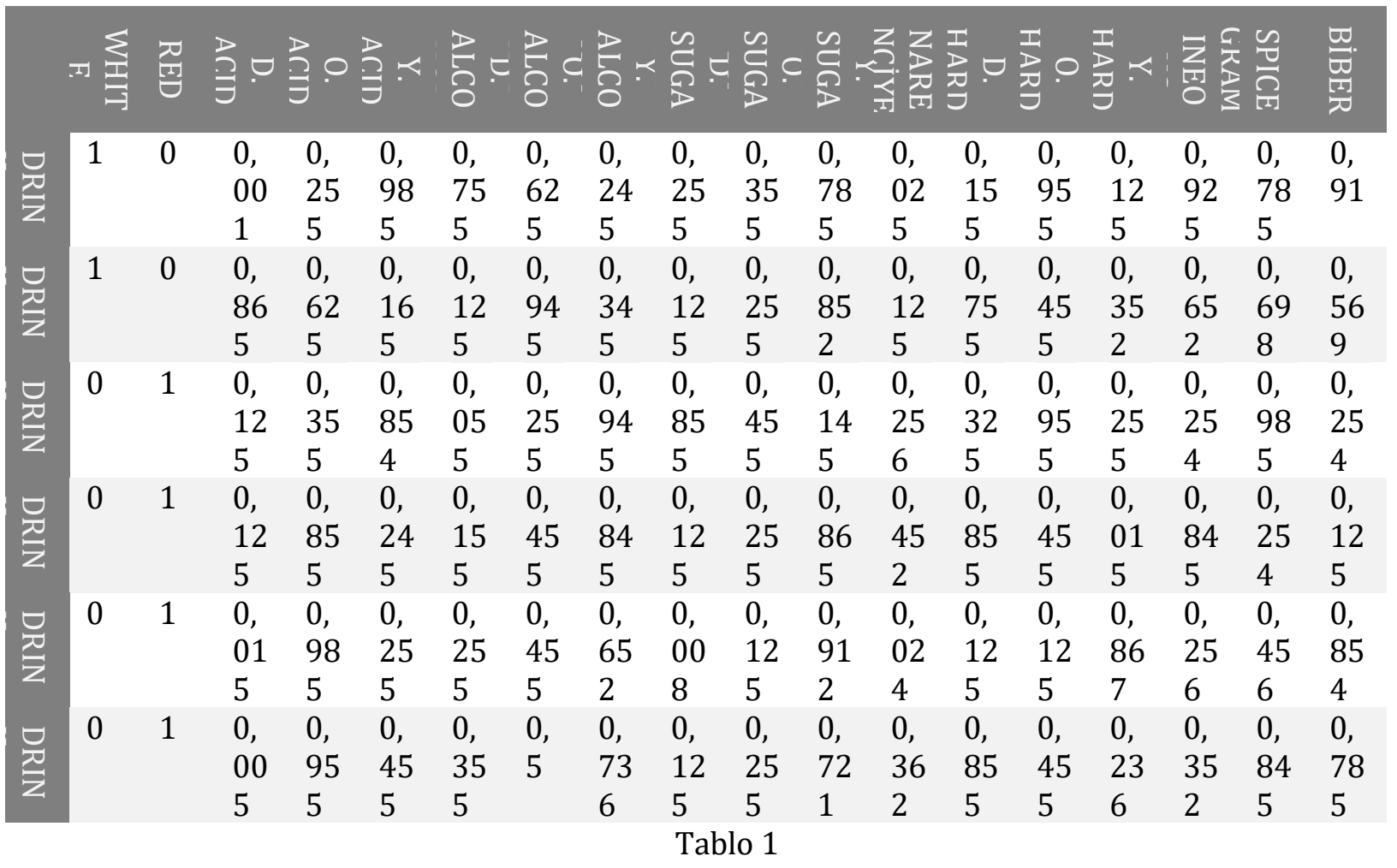

\begin{tabular}{|c|c|c|c|c|c|c|c|c|c|c|c|c|}
\hline & 㞼西 & 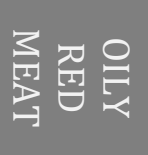 & 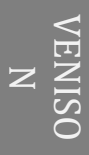 & z $\underset{\bar{I}}{\text { 皇 }}$ & $\begin{array}{l}\text { 包 } \\
\text { 尝 } \\
\text { 焉 }\end{array}$ & $\begin{array}{l}\frac{\pi}{4} \\
\text { 吾 }\end{array}$ & 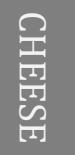 & $\stackrel{\text { 경 }}{\subseteq}$ & 四 & 끚 & $\frac{\sqrt[n]{3}}{\frac{3}{3}}$ & $\frac{n}{\overparen{7}}$ \\
\hline \multirow[t]{2}{*}{ WHITE } & 0,24 & 0,015 & 0,1 & 0,85 & 0,952 & 0,89 & 0,8 & 0,7 & 0,8 & 0,865 & 0,8 & 0,84 \\
\hline & 5 & & 5 & 3 & & 5 & 5 & 85 & 25 & & 45 & 5 \\
\hline \multirow[t]{2}{*}{ RED } & 0,85 & 0,855 & 0,9 & 0,55 & 0,245 & 0,85 & 0,9 & 0,6 & 0,1 & 0,525 & 0,4 & 0,85 \\
\hline & 5 & & 25 & 5 & & 5 & 55 & 55 & 5 & & 25 & 2 \\
\hline \multirow[t]{2}{*}{ D. ACID } & 0,01 & 0,015 & 0,0 & 0,12 & 0,015 & 0,32 & 0,2 & 0,2 & 0,4 & 0,225 & 0,2 & 0,22 \\
\hline & 5 & & 15 & 5 & & 5 & 25 & 45 & 55 & & 25 & 5 \\
\hline \multirow[t]{2}{*}{ O. ACID } & 0,22 & 0,225 & 0,2 & 0,42 & 0,225 & 0,55 & 0,4 & 0,5 & 0,3 & 0,425 & 0,4 & 0,42 \\
\hline & 5 & & 25 & 5 & & 5 & 25 & 85 & 25 & & 25 & 5 \\
\hline \multirow[t]{2}{*}{ Y. ACID } & 0,95 & 0,952 & 0,9 & 0,85 & 0,952 & 0,75 & 0,8 & 0,7 & 0,0 & 0,865 & 0,8 & 0,84 \\
\hline & 2 & & 52 & 3 & & & 5 & 85 & 15 & & 45 & 5 \\
\hline D. & 0,12 & 0,015 & 0,0 & 0,62 & 0,625 & 0,62 & 0,5 & 0,6 & 0,5 & 0,015 & 0,6 & 0,12 \\
\hline ALCOHOL & 5 & & 15 & 5 & & 5 & 45 & 25 & 25 & & 25 & 5 \\
\hline & 0,42 & 0,225 & 0,2 & 0,42 & 0,425 & 0,42 & 0,4 & 0,4 & 0,1 & 0,225 & 0,4 & 0,42 \\
\hline ALCOHOL & 5 & & 25 & 5 & & 5 & 5 & 25 & 25 & & 25 & 5 \\
\hline
\end{tabular}




$\begin{array}{lllllllllllll}\text { Y. } & 0,85 & 0,952 & 0,9 & 0,12 & 0,125 & 0,12 & 0,7 & 0,1 & 0,1 & 0,952 & 0,1 & 0,85 \\ \text { ALCOHOL } & 3 & & 52 & 5 & & 5 & 85 & 25 & 5 & & 25 & 3 \\ \text { D. SUGARY } & 0,74 & 0,525 & 0,5 & 0,32 & 0,325 & 0,32 & 0,8 & 0,8 & 0,4 & 0,425 & 0,3 & 0,82 \\ & 5 & & 25 & 5 & & 5 & 25 & 45 & 25 & & 25 & 5 \\ \text { O. SUGARY } & 0,52 & 0,325 & 0,3 & 0,82 & 0,825 & 0,82 & 0,4 & 0,5 & 0,1 & 0,15 & 0,8 & 0,42 \\ & 5 & & 25 & 5 & & 5 & 55 & 45 & 25 & & 45 & 5 \\ \text { Y. SUGARY } & 0,12 & 0,125 & 0,1 & 0,45 & 0,495 & 0,85 & 0,3 & 0,4 & 0,1 & 0,015 & 0,2 & 0,35 \\ & 5 & & 25 & 5 & & 5 & 5 & 5 & 5 & & 55 & 5 \\ \text { NARENCIY } & 0,35 & 0,55 & 0,5 & 0,85 & 0,952 & 0,89 & 0,8 & 0,7 & 0,4 & 0,865 & 0,8 & 0,84 \\ \text { E } & & & 5 & 3 & & 5 & 5 & 85 & 25 & & 45 & 5 \\ \text { D. HARD } & 0,12 & 0,125 & 0,1 & 0,12 & 0,125 & 0,12 & 0,2 & 0,1 & 0,4 & 0,125 & 0,1 & 0,54 \\ & 5 & & 25 & 5 & & 5 & 25 & 25 & 25 & & 25 & 5 \\ \text { O. HARD } & 0,25 & 0,425 & 0,4 & 0,25 & 0,255 & 0,74 & 0,4 & 0,3 & 0,1 & 0,255 & 0,2 & 0,34 \\ & 5 & & 25 & 5 & & 5 & 55 & 45 & 25 & & 55 & 5 \\ \text { Y. HARD } & 0,85 & 0,753 & 0,7 & 0,95 & 0,955 & 0,12 & 0,7 & 0,8 & 0,0 & 0,855 & 0,8 & 0,24 \\ & 5 & & 53 & 2 & & 5 & 5 & 5 & 15 & & 5 & 5 \\ \text { GRAMINE } & 0,42 & 0,75 & 0,7 & 0,85 & 0,952 & 0,89 & 0,8 & 0,7 & 0,9 & 0,865 & 0,8 & 0,84 \\ \text { OUS } & 5 & & 5 & 3 & & 5 & 5 & 85 & 25 & & 45 & 5 \\ \text { SPICE } & 0,75 & 0,75 & 0,8 & 0,74 & 0,745 & 0,85 & 0,9 & 0,1 & 0,8 & 0,95 & 0,7 & 0,56 \\ & 5 & & 9 & 5 & & & 85 & 25 & 55 & & 25 & \\ \text { RED FRUIT } & 0,75 & 0,55 & 0,4 & 0,85 & 0,745 & 0,85 & 0,8 & 0,1 & 0,2 & 0,125 & 0,1 & 0,32 \\ & & & 55 & 5 & & 4 & 55 & 25 & 55 & & 25 & 5 \\ \text { PIPER } & 0,85 & 0,865 & 0,9 & 0,84 & 0,845 & 0,75 & 0,9 & 0,1 & 0,7 & 0,95 & 0,8 & 0,12 \\ & 5 & & 85 & 5 & & 2 & 85 & 25 & 55 & & 25 & 5 \\ \text { FRUIT } & 0,54 & 0,325 & 0,2 & 0,32 & 0,325 & 0,12 & 0,7 & 0,1 & 0,1 & 0,526 & 0 & 0,12 \\ \text { FLAVORS } & 6 & & 56 & 5 & & 5 & 25 & 25 & 25 & & & 5\end{array}$

\subsection{Data Structure}

The Microsoft SQL Server data structure is created as follows.

Beverage Products Chart

Food Types Chart

Table of features of the product

CREATE TABLE [dbo].[TBL_PROPERTY](

[ID] [bigint] IDENTITY $(1,1)$ NOT NULL,

[DESCRIPTION] [nvarchar](50) NULL,

CONSTRAINT [PK_TBL_PROPERTY] PRIMARY KEY CLUSTERED

[ID] ASC

)WITH (PAD_INDEX $=$ OFF, STATISTICS_NORECOMPUTE $=$ OFF, IGNORE_DUP_KEY $=$ OFF,

ALLOW_ROW_LOCKS $=$ ON, ALLOW_PAGE_LOCKS $=$ ON) ON [PRIMARY]

) ON [PRIMARY] 


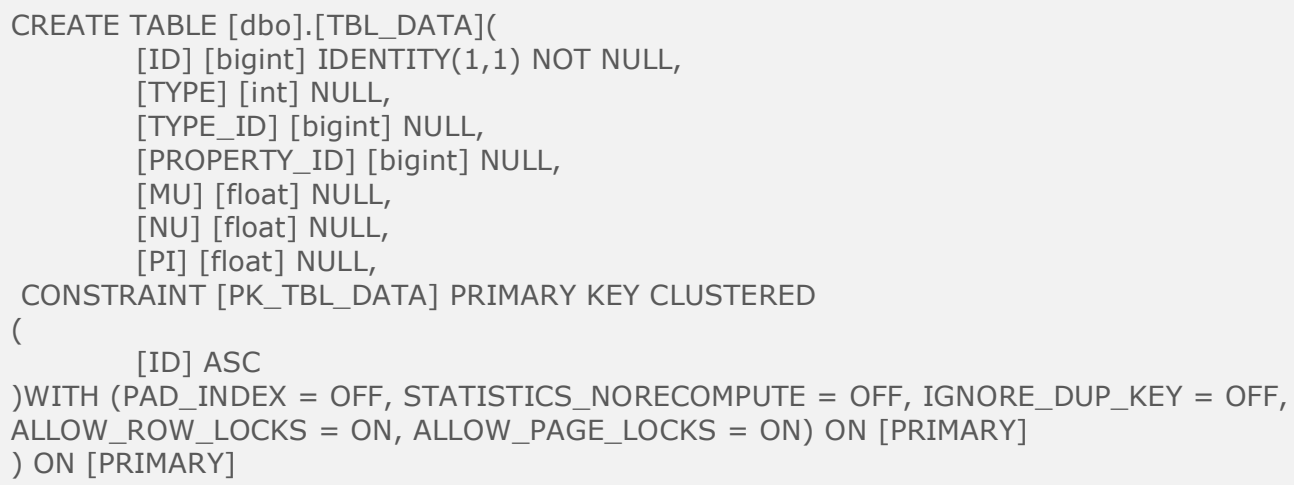

Veri Tablosu

\subsection{Data Arrangement}

Data entry on Microsoft SQL Server is done as followsTBL_DRINK

\begin{tabular}{|ll}
\hline ID & DESCRIPTION \\
\hline 1 & DRINK NO.1 \\
2 & DRINK NO.2 \\
3 & DRINK NO.3 \\
4 & DRINK NO.4 \\
5 & DRINK NO.5 \\
6 & DRINK NO.6 \\
\hline
\end{tabular}

Table-3

TBL_FOOD

$\begin{array}{ll}\text { ID } & \text { DESCRIPTION } \\ 1 & \text { RED MEAT } \\ 2 & \text { OILY RED MEAT } \\ 3 & \text { VENISON } \\ 4 & \text { CHICKEN } \\ 5 & \text { TURKEY } \\ 6 & \text { FISH } \\ 7 & \text { CHEESE } \\ 8 & \text { FRUIT } \\ 9 & \text { VEGETABLES } \\ 10 & \text { PORK } \\ 11 & \text { SHRIMP } \\ 12 & \text { SPICE }\end{array}$

Table-4

TBL_PROPERTY 


\begin{tabular}{ll}
\hline ID & DESCRIPTION \\
\hline $\mathbf{1}$ & RED \\
$\mathbf{2}$ & WHITE \\
$\mathbf{3}$ & ACIDITYL \\
$\mathbf{4}$ & ACIDITYM \\
$\mathbf{5}$ & ACIDITYH \\
$\mathbf{6}$ & ALCOHOLL \\
$\mathbf{7}$ & ALCOHOLM \\
$\mathbf{8}$ & ALCOHOLH \\
$\mathbf{9}$ & SWEETL \\
10 & SWEETM \\
11 & SWEETH \\
12 & CITRUS \\
\hline
\end{tabular}

Table-5

Each value that gives the relationship between the elements of the A-C and C-S sets is determined by the degree of membership, degree of non-membership, and degree of intuition.

The $\mathrm{C}$ property of each element of $\mathrm{A}$ is entered one by one.

$S$ relation of each element of $C$ is entered one by one.

TBL_DATA

$\begin{array}{lllll}\text { ID } & \text { TYPE } & \text { TYPE_ID } & \text { PROPERTY_ID } & \text { MU } \\ 2 & 1 & 1 & 1 & 0 \\ 3 & 1 & 1 & 2 & 1 \\ 4 & 1 & 1 & 3 & 0,001 \\ 5 & 1 & 1 & 4 & 0,255 \\ 6 & 1 & 1 & 5 & 0,985 \\ 7 & 1 & 1 & 6 & 0,755 \\ 8 & 1 & 1 & 7 & 0,625 \\ 9 & 1 & 1 & 8 & 0,245 \\ 10 & 1 & 1 & 9 & 0,255 \\ 11 & 1 & 1 & 10 & 0,355 \\ 12 & 1 & 1 & 11 & 0,785 \\ 13 & 1 & 1 & 12 & 0,025 \\ 14 & 1 & 1 & 13 & 0,155 \\ 15 & 1 & 1 & 14 & 0,955 \\ 16 & 1 & 1 & 15 & 0,125 \\ 17 & 1 & 1 & 16 & 0,925 \\ 18 & 1 & 1 & 17 & 0,785 \\ 19 & 1 & 1 & 18 & 0,001 \\ 20 & 1 & 1 & 19 & 0,91 \\ 21 & 1 & 1 & 20 & 0,895 \\ 22 & 1 & 2 & 1 & 0 \\ 23 & 1 & 2 & 2 & 1 \\ 24 & 1 & 2 & 3 & 0,865 \\ 25 & 1 & 2 & 4 & 0,625 \\ 26 & 1 & 2 & 5 & 0,165\end{array}$




\begin{tabular}{|c|c|c|c|c|}
\hline 27 & 1 & 2 & 6 & 0,125 \\
\hline 28 & 1 & 2 & 7 & 0,945 \\
\hline 29 & 1 & 2 & 8 & 0,345 \\
\hline 30 & 1 & 2 & 9 & 0,125 \\
\hline 31 & 1 & 2 & 10 & 0,255 \\
\hline 32 & 1 & 2 & 11 & 0,852 \\
\hline 33 & 1 & 2 & 12 & 0,125 \\
\hline 34 & 1 & 2 & 13 & 0,755 \\
\hline 35 & 1 & 2 & 14 & 0,455 \\
\hline 36 & 1 & 2 & 15 & 0,352 \\
\hline 37 & 1 & 2 & 16 & 0,652 \\
\hline 38 & 1 & 2 & 17 & 0,698 \\
\hline 39 & 1 & 2 & 18 & 0,021 \\
\hline 40 & 1 & 2 & 19 & 0,569 \\
\hline 41 & 1 & 2 & 20 & 0,825 \\
\hline 42 & 1 & 3 & 1 & 1 \\
\hline 43 & 1 & 3 & 2 & 0 \\
\hline 44 & 1 & 3 & 3 & 0,125 \\
\hline 45 & 1 & 3 & 4 & 0,355 \\
\hline 46 & 1 & 3 & 5 & 0,854 \\
\hline 47 & 1 & 3 & 6 & 0,055 \\
\hline 48 & 1 & 3 & 7 & 0,255 \\
\hline 49 & 1 & 3 & 8 & 0,945 \\
\hline 50 & 1 & 3 & 9 & 0,855 \\
\hline 51 & 1 & 3 & 10 & 0,455 \\
\hline 52 & 1 & 3 & 11 & 0,145 \\
\hline 53 & 1 & 3 & 12 & 0,256 \\
\hline 54 & 1 & 3 & 13 & 0,325 \\
\hline 55 & 1 & 3 & 14 & 0,955 \\
\hline 56 & 1 & 3 & 15 & 0,255 \\
\hline 57 & 1 & 3 & 16 & 0,254 \\
\hline 58 & 1 & 3 & 17 & 0,985 \\
\hline 59 & 1 & 3 & 18 & 0,985 \\
\hline 60 & 1 & 3 & 19 & 0,254 \\
\hline 61 & 1 & 3 & 20 & 0,785 \\
\hline 62 & 1 & 4 & 1 & 1 \\
\hline 63 & 1 & 4 & 2 & 0 \\
\hline 64 & 1 & 4 & 3 & 0,125 \\
\hline 65 & 1 & 4 & 4 & 0,855 \\
\hline 66 & 1 & 4 & 5 & 0,245 \\
\hline 67 & 1 & 4 & 6 & 0,155 \\
\hline 68 & 1 & 4 & 7 & 0,455 \\
\hline 69 & 1 & 4 & 8 & 0,845 \\
\hline 70 & 1 & 4 & 9 & 0,125 \\
\hline 71 & 1 & 4 & 10 & 0,255 \\
\hline 72 & 1 & 4 & 11 & 0,865 \\
\hline 73 & 1 & 4 & 12 & 0,452 \\
\hline 74 & 1 & 4 & 13 & 0,855 \\
\hline 75 & 1 & 4 & 14 & 0,455 \\
\hline
\end{tabular}




\begin{tabular}{|c|c|c|c|c|}
\hline 76 & 1 & 4 & 15 & 0,015 \\
\hline 77 & 1 & 4 & 16 & 0,845 \\
\hline 78 & 1 & 4 & 17 & 0,254 \\
\hline 79 & 1 & 4 & 18 & 0,654 \\
\hline 80 & 1 & 4 & 19 & 0,125 \\
\hline 81 & 1 & 4 & 20 & 0,645 \\
\hline 82 & 1 & 5 & 1 & 1 \\
\hline 83 & 1 & 5 & 2 & 0 \\
\hline 84 & 1 & 5 & 3 & 0,015 \\
\hline 85 & 1 & 5 & 4 & 0,985 \\
\hline 86 & 1 & 5 & 5 & 0,255 \\
\hline 87 & 1 & 5 & 6 & 0,255 \\
\hline 88 & 1 & 5 & 7 & 0,455 \\
\hline 89 & 1 & 5 & 8 & 0,652 \\
\hline 90 & 1 & 5 & 9 & 0,008 \\
\hline 91 & 1 & 5 & 10 & 0,125 \\
\hline 92 & 1 & 5 & 11 & 0,912 \\
\hline 93 & 1 & 5 & 12 & 0,024 \\
\hline 94 & 1 & 5 & 13 & 0,125 \\
\hline 95 & 1 & 5 & 14 & 0,125 \\
\hline 96 & 1 & 5 & 15 & 0,867 \\
\hline 97 & 1 & 5 & 16 & 0,256 \\
\hline 98 & 1 & 5 & 17 & 0,456 \\
\hline 99 & 1 & 5 & 18 & 0,985 \\
\hline 100 & 1 & 5 & 19 & 0,854 \\
\hline 101 & 1 & 5 & 20 & 0,856 \\
\hline 102 & 1 & 6 & 1 & 1 \\
\hline 103 & 1 & 6 & 2 & 0 \\
\hline 104 & 1 & 6 & 3 & 0,005 \\
\hline 105 & 1 & 6 & 4 & 0,955 \\
\hline 106 & 1 & 6 & 5 & 0,455 \\
\hline 107 & 1 & 6 & 6 & 0,355 \\
\hline 108 & 1 & 6 & 7 & 0,5 \\
\hline 109 & 1 & 6 & 8 & 0,736 \\
\hline 110 & 1 & 6 & 9 & 0,125 \\
\hline 111 & 1 & 6 & 10 & 0,255 \\
\hline 112 & 1 & 6 & 11 & 0,721 \\
\hline 113 & 1 & 6 & 12 & 0,362 \\
\hline 114 & 1 & 6 & 13 & 0,855 \\
\hline 115 & 1 & 6 & 14 & 0,455 \\
\hline 116 & 1 & 6 & 15 & 0,236 \\
\hline 117 & 1 & 6 & 16 & 0,352 \\
\hline 118 & 1 & 6 & 17 & 0,845 \\
\hline 119 & 1 & 6 & 18 & 0,995 \\
\hline 120 & 1 & 6 & 19 & 0,785 \\
\hline 121 & 1 & 6 & 20 & 0,886 \\
\hline 122 & 2 & 1 & 1 & 0,855 \\
\hline 123 & 2 & 1 & 2 & 0,245 \\
\hline 124 & 2 & 1 & 3 & 0,015 \\
\hline
\end{tabular}




\begin{tabular}{|c|c|c|c|c|}
\hline 125 & 2 & 1 & 4 & 0,225 \\
\hline 126 & 2 & 1 & 5 & 0,952 \\
\hline 127 & 2 & 1 & 6 & 0,125 \\
\hline 128 & 2 & 1 & 7 & 0,425 \\
\hline 129 & 2 & 1 & 8 & 0,853 \\
\hline 130 & 2 & 1 & 9 & 0,745 \\
\hline 131 & 2 & 1 & 10 & 0,525 \\
\hline 132 & 2 & 1 & 11 & 0,125 \\
\hline 133 & 2 & 1 & 12 & 0,35 \\
\hline 134 & 2 & 1 & 13 & 0,125 \\
\hline 135 & 2 & 1 & 14 & 0,255 \\
\hline 136 & 2 & 1 & 15 & 0,855 \\
\hline 137 & 2 & 1 & 16 & 0,425 \\
\hline 138 & 2 & 1 & 17 & 0,755 \\
\hline 139 & 2 & 1 & 18 & 0,75 \\
\hline 140 & 2 & 1 & 19 & 0,855 \\
\hline 141 & 2 & 1 & 20 & 0,546 \\
\hline 142 & 2 & 2 & 1 & 0,855 \\
\hline 143 & 2 & 2 & 2 & 0,015 \\
\hline 144 & 2 & 2 & 3 & 0,015 \\
\hline 145 & 2 & 2 & 4 & 0,225 \\
\hline 146 & 2 & 2 & 5 & 0,952 \\
\hline 147 & 2 & 2 & 6 & 0,015 \\
\hline 148 & 2 & 2 & 7 & 0,225 \\
\hline 149 & 2 & 2 & 8 & 0,952 \\
\hline 150 & 2 & 2 & 9 & 0,525 \\
\hline 151 & 2 & 2 & 10 & 0,325 \\
\hline 152 & 2 & 2 & 11 & 0,125 \\
\hline 153 & 2 & 2 & 12 & 0,55 \\
\hline 154 & 2 & 2 & 13 & 0,125 \\
\hline 155 & 2 & 2 & 14 & 0,425 \\
\hline 156 & 2 & 2 & 15 & 0,753 \\
\hline 157 & 2 & 2 & 16 & 0,75 \\
\hline 158 & 2 & 2 & 17 & 0,75 \\
\hline 159 & 2 & 2 & 18 & 0,55 \\
\hline 160 & 2 & 2 & 19 & 0,865 \\
\hline 161 & 2 & 2 & 20 & 0,325 \\
\hline 162 & 2 & 3 & 1 & 0,925 \\
\hline 163 & 2 & 3 & 2 & 0,15 \\
\hline 164 & 2 & 3 & 3 & 0,015 \\
\hline 165 & 2 & 3 & 4 & 0,225 \\
\hline 166 & 2 & 3 & 5 & 0,952 \\
\hline 167 & 2 & 3 & 6 & 0,015 \\
\hline 168 & 2 & 3 & 7 & 0,225 \\
\hline 169 & 2 & 3 & 8 & 0,952 \\
\hline 170 & 2 & 3 & 9 & 0,525 \\
\hline 171 & 2 & 3 & 10 & 0,325 \\
\hline 172 & 2 & 3 & 11 & 0,125 \\
\hline 173 & 2 & 3 & 12 & 0,55 \\
\hline
\end{tabular}




\begin{tabular}{|c|c|c|c|c|}
\hline 174 & 2 & 3 & 13 & 0,125 \\
\hline 175 & 2 & 3 & 14 & 0,425 \\
\hline 176 & 2 & 3 & 15 & 0,753 \\
\hline 177 & 2 & 3 & 16 & 0,75 \\
\hline 178 & 2 & 3 & 17 & 0,89 \\
\hline 179 & 2 & 3 & 18 & 0,455 \\
\hline 180 & 2 & 3 & 19 & 0,985 \\
\hline 181 & 2 & 3 & 20 & 0,256 \\
\hline 182 & 2 & 4 & 1 & 0,555 \\
\hline 183 & 2 & 4 & 2 & 0,853 \\
\hline 184 & 2 & 4 & 3 & 0,125 \\
\hline 185 & 2 & 4 & 4 & 0,425 \\
\hline 186 & 2 & 4 & 5 & 0,853 \\
\hline 187 & 2 & 4 & 6 & 0,625 \\
\hline 188 & 2 & 4 & 7 & 0,425 \\
\hline 189 & 2 & 4 & 8 & 0,125 \\
\hline 190 & 2 & 4 & 9 & 0,325 \\
\hline 191 & 2 & 4 & 10 & 0,825 \\
\hline 192 & 2 & 4 & 11 & 0,455 \\
\hline 193 & 2 & 4 & 12 & 0,853 \\
\hline 194 & 2 & 4 & 13 & 0,125 \\
\hline 195 & 2 & 4 & 14 & 0,255 \\
\hline 196 & 2 & 4 & 15 & 0,952 \\
\hline 197 & 2 & 4 & 16 & 0,853 \\
\hline 198 & 2 & 4 & 17 & 0,745 \\
\hline 199 & 2 & 4 & 18 & 0,855 \\
\hline 200 & 2 & 4 & 19 & 0,845 \\
\hline 201 & 2 & 4 & 20 & 0,325 \\
\hline 202 & 2 & 5 & 1 & 0,245 \\
\hline 203 & 2 & 5 & 2 & 0,952 \\
\hline 204 & 2 & 5 & 3 & 0,015 \\
\hline 205 & 2 & 5 & 4 & 0,225 \\
\hline 206 & 2 & 5 & 5 & 0,952 \\
\hline 207 & 2 & 5 & 6 & 0,625 \\
\hline 208 & 2 & 5 & 7 & 0,425 \\
\hline 209 & 2 & 5 & 8 & 0,125 \\
\hline 210 & 2 & 5 & 9 & 0,325 \\
\hline 211 & 2 & 5 & 10 & 0,825 \\
\hline 212 & 2 & 5 & 11 & 0,495 \\
\hline 213 & 2 & 5 & 12 & 0,952 \\
\hline 214 & 2 & 5 & 13 & 0,125 \\
\hline 215 & 2 & 5 & 14 & 0,255 \\
\hline 216 & 2 & 5 & 15 & 0,955 \\
\hline 217 & 2 & 5 & 16 & 0,952 \\
\hline 218 & 2 & 5 & 17 & 0,745 \\
\hline 219 & 2 & 5 & 18 & 0,745 \\
\hline 220 & 2 & 5 & 19 & 0,845 \\
\hline 221 & 2 & 5 & 20 & 0,325 \\
\hline 222 & 2 & 6 & 1 & 0,855 \\
\hline
\end{tabular}




\begin{tabular}{|c|c|c|c|c|}
\hline 223 & 2 & 6 & 2 & 0,895 \\
\hline 224 & 2 & 6 & 3 & 0,325 \\
\hline 225 & 2 & 6 & 4 & 0,555 \\
\hline 226 & 2 & 6 & 5 & 0,75 \\
\hline 227 & 2 & 6 & 6 & 0,625 \\
\hline 228 & 2 & 6 & 7 & 0,425 \\
\hline 229 & 2 & 6 & 8 & 0,125 \\
\hline 230 & 2 & 6 & 9 & 0,325 \\
\hline 231 & 2 & 6 & 10 & 0,825 \\
\hline 232 & 2 & 6 & 11 & 0,855 \\
\hline 233 & 2 & 6 & 12 & 0,895 \\
\hline 234 & 2 & 6 & 13 & 0,125 \\
\hline 235 & 2 & 6 & 14 & 0,745 \\
\hline 236 & 2 & 6 & 15 & 0,125 \\
\hline 237 & 2 & 6 & 16 & 0,895 \\
\hline 238 & 2 & 6 & 17 & 0,85 \\
\hline 239 & 2 & 6 & 18 & 0,854 \\
\hline 240 & 2 & 6 & 19 & 0,752 \\
\hline 241 & 2 & 6 & 20 & 0,125 \\
\hline 242 & 2 & 7 & 1 & 0,955 \\
\hline 243 & 2 & 7 & 2 & 0,85 \\
\hline 244 & 2 & 7 & 3 & 0,225 \\
\hline 245 & 2 & 7 & 4 & 0,425 \\
\hline 246 & 2 & 7 & 5 & 0,85 \\
\hline 247 & 2 & 7 & 6 & 0,545 \\
\hline 248 & 2 & 7 & 7 & 0,45 \\
\hline 249 & 2 & 7 & 8 & 0,785 \\
\hline 250 & 2 & 7 & 9 & 0,825 \\
\hline 251 & 2 & 7 & 10 & 0,455 \\
\hline 252 & 2 & 7 & 11 & 0,35 \\
\hline 253 & 2 & 7 & 12 & 0,85 \\
\hline 254 & 2 & 7 & 13 & 0,225 \\
\hline 255 & 2 & 7 & 14 & 0,455 \\
\hline 256 & 2 & 7 & 15 & 0,75 \\
\hline 257 & 2 & 7 & 16 & 0,85 \\
\hline 258 & 2 & 7 & 17 & 0,985 \\
\hline 259 & 2 & 7 & 18 & 0,855 \\
\hline 260 & 2 & 7 & 19 & 0,985 \\
\hline 261 & 2 & 7 & 20 & 0,725 \\
\hline 262 & 2 & 8 & 1 & 0,655 \\
\hline 263 & 2 & 8 & 2 & 0,785 \\
\hline 264 & 2 & 8 & 3 & 0,245 \\
\hline 265 & 2 & 8 & 4 & 0,585 \\
\hline 266 & 2 & 8 & 5 & 0,785 \\
\hline 267 & 2 & 8 & 6 & 0,625 \\
\hline 268 & 2 & 8 & 7 & 0,425 \\
\hline 269 & 2 & 8 & 8 & 0,125 \\
\hline 270 & 2 & 8 & 9 & 0,845 \\
\hline 271 & 2 & 8 & 10 & 0,545 \\
\hline
\end{tabular}




\begin{tabular}{|c|c|c|c|c|}
\hline 272 & 2 & 8 & 11 & 0,45 \\
\hline 273 & 2 & 8 & 12 & 0,785 \\
\hline 274 & 2 & 8 & 13 & 0,125 \\
\hline 275 & 2 & 8 & 14 & 0,345 \\
\hline 276 & 2 & 8 & 15 & 0,85 \\
\hline 277 & 2 & 8 & 16 & 0,785 \\
\hline 278 & 2 & 8 & 17 & 0,125 \\
\hline 279 & 2 & 8 & 18 & 0,125 \\
\hline 280 & 2 & 8 & 19 & 0,125 \\
\hline 281 & 2 & 8 & 20 & 0,125 \\
\hline 282 & 2 & 9 & 1 & 0,15 \\
\hline 283 & 2 & 9 & 2 & 0,825 \\
\hline 284 & 2 & 9 & 3 & 0,455 \\
\hline 285 & 2 & 9 & 4 & 0,325 \\
\hline 286 & 2 & 9 & 5 & 0,015 \\
\hline 287 & 2 & 9 & 6 & 0,525 \\
\hline 288 & 2 & 9 & 7 & 0,125 \\
\hline 289 & 2 & 9 & 8 & 0,15 \\
\hline 290 & 2 & 9 & 9 & 0,425 \\
\hline 291 & 2 & 9 & 10 & 0,125 \\
\hline 292 & 2 & 9 & 11 & 0,15 \\
\hline 293 & 2 & 9 & 12 & 0,425 \\
\hline 294 & 2 & 9 & 13 & 0,425 \\
\hline 295 & 2 & 9 & 14 & 0,125 \\
\hline 296 & 2 & 9 & 15 & 0,015 \\
\hline 297 & 2 & 9 & 16 & 0,925 \\
\hline 298 & 2 & 9 & 17 & 0,855 \\
\hline 299 & 2 & 9 & 18 & 0,255 \\
\hline 300 & 2 & 9 & 19 & 0,755 \\
\hline 301 & 2 & 9 & 20 & 0,125 \\
\hline 302 & 2 & 10 & 1 & 0,525 \\
\hline 303 & 2 & 10 & 2 & 0,865 \\
\hline 304 & 2 & 10 & 3 & 0,225 \\
\hline 305 & 2 & 10 & 4 & 0,425 \\
\hline 306 & 2 & 10 & 5 & 0,865 \\
\hline 307 & 2 & 10 & 6 & 0,015 \\
\hline 308 & 2 & 10 & 7 & 0,225 \\
\hline 309 & 2 & 10 & 8 & 0,952 \\
\hline 310 & 2 & 10 & 9 & 0,425 \\
\hline 311 & 2 & 10 & 10 & 0,15 \\
\hline 312 & 2 & 10 & 11 & 0,015 \\
\hline 313 & 2 & 10 & 12 & 0,865 \\
\hline 314 & 2 & 10 & 13 & 0,125 \\
\hline 315 & 2 & 10 & 14 & 0,255 \\
\hline 316 & 2 & 10 & 15 & 0,855 \\
\hline 317 & 2 & 10 & 16 & 0,865 \\
\hline 318 & 2 & 10 & 17 & 0,95 \\
\hline 319 & 2 & 10 & 18 & 0,125 \\
\hline 320 & 2 & 10 & 19 & 0,95 \\
\hline
\end{tabular}




\begin{tabular}{|c|c|c|c|c|}
\hline 321 & 2 & 10 & 20 & 0,526 \\
\hline 322 & 2 & 11 & 1 & 0,425 \\
\hline 323 & 2 & 11 & 2 & 0,845 \\
\hline 324 & 2 & 11 & 3 & 0,225 \\
\hline 325 & 2 & 11 & 4 & 0,425 \\
\hline 326 & 2 & 11 & 5 & 0,845 \\
\hline 327 & 2 & 11 & 6 & 0,625 \\
\hline 328 & 2 & 11 & 7 & 0,425 \\
\hline 329 & 2 & 11 & 8 & 0,125 \\
\hline 330 & 2 & 11 & 9 & 0,325 \\
\hline 331 & 2 & 11 & 10 & 0,845 \\
\hline 332 & 2 & 11 & 11 & 0,255 \\
\hline 333 & 2 & 11 & 12 & 0,845 \\
\hline 334 & 2 & 11 & 13 & 0,125 \\
\hline 335 & 2 & 11 & 14 & 0,255 \\
\hline 336 & 2 & 11 & 15 & 0,85 \\
\hline 337 & 2 & 11 & 16 & 0,845 \\
\hline 338 & 2 & 11 & 17 & 0,725 \\
\hline 339 & 2 & 11 & 18 & 0,125 \\
\hline 340 & 2 & 11 & 19 & 0,825 \\
\hline 341 & 2 & 11 & 20 & 0 \\
\hline 342 & 2 & 12 & 1 & 0,852 \\
\hline 343 & 2 & 12 & 2 & 0,845 \\
\hline 344 & 2 & 12 & 3 & 0,225 \\
\hline 345 & 2 & 12 & 4 & 0,425 \\
\hline 346 & 2 & 12 & 5 & 0,845 \\
\hline 347 & 2 & 12 & 6 & 0,125 \\
\hline 348 & 2 & 12 & 7 & 0,425 \\
\hline 349 & 2 & 12 & 8 & 0,853 \\
\hline 350 & 2 & 12 & 9 & 0,825 \\
\hline 351 & 2 & 12 & 10 & 0,425 \\
\hline 352 & 2 & 12 & 11 & 0,355 \\
\hline 353 & 2 & 12 & 12 & 0,845 \\
\hline 354 & 2 & 12 & 13 & 0,545 \\
\hline 355 & 2 & 12 & 14 & 0,345 \\
\hline 356 & 2 & 12 & 15 & 0,245 \\
\hline 357 & 2 & 12 & 16 & 0,845 \\
\hline 358 & 2 & 12 & 17 & 0,56 \\
\hline 359 & 2 & 12 & 18 & 0,325 \\
\hline 360 & 2 & 12 & 19 & 0,125 \\
\hline 361 & 2 & 12 & 20 & 0,125 \\
\hline
\end{tabular}


These values are as in Table- 1 and Table- 2 and as in Table-6, "TYPE=1" Beverage Products, "TYPE=2" Food Types are arranged with TYPE_ID connections on Microsoft SQL Server.

By using the controlled set feature, these values are controlled through Description-1 and Description-2.

$\begin{array}{llllll}\text { TYPE } & \text { TYPE_ID } & \text { PROPERTY_ID } & \text { MU } & \text { NU } & \text { PI } \\ \mathbf{1} & 2 & 15 & 0,352 & 0,352 & 0,296 \\ \mathbf{1} & 5 & 15 & 0,867 & 0,125 & 0,008 \\ \mathbf{1} & 1 & 17 & 0,785 & 0 & 0,215 \\ \mathbf{1} & 2 & 10 & 0,255 & 0,455 & 0,29 \\ \mathbf{1} & 6 & 15 & 0,236 & 0,352 & 0,412 \\ \mathbf{2} & 10 & 19 & 0,95 & 0 & 0,05 \\ \mathbf{1} & 3 & 18 & 0,985 & 0,001 & 0,014 \\ 1 & 2 & 5 & 0,165 & 0,455 & 0,38 \\ \mathbf{2} & 3 & 17 & 0,89 & 0 & 0,11 \\ \mathbf{2} & 12 & 15 & 0,245 & 0,753 & 0,002 \\ \mathbf{2} & 8 & 4 & 0,585 & 0,325 & 0,09 \\ \mathbf{2} & 6 & 20 & 0,125 & 0,725 & 0,15 \\ \mathbf{2} & 9 & 4 & 0,325 & 0,585 & 0,09\end{array}$

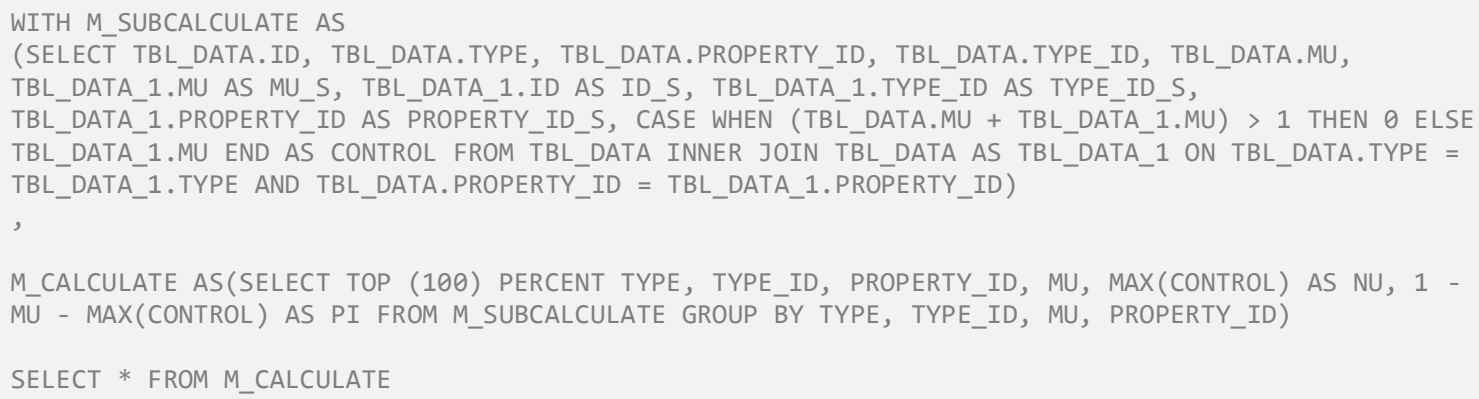

$\begin{array}{llllll}2 & 4 & 20 & 0,325 & 0,546 & 0,129 \\ 1 & 4 & 9 & 0,125 & 0,855 & 0,02 \\ 2 & 9 & 2 & 0,825 & 0,15 & 0,025 \\ 2 & 11 & 5 & 0,845 & 0,015 & 0,14 \\ 1 & 3 & 7 & 0,255 & 0,625 & 0,12 \\ 2 & 5 & 16 & 0,952 & 0 & 0,048 \\ 2 & 12 & 2 & 0,845 & 0,15 & 0,005 \\ 2 & 10 & 20 & 0,526 & 0,325 & 0,149 \\ 2 & 11 & 2 & 0,845 & 0,15 & 0,005 \\ 1 & 6 & 4 & 0,955 & 0 & 0,045 \\ 2 & 9 & 8 & 0,15 & 0,785 & 0,065 \\ 1 & 1 & 1 & 0 & 1 & 0 \\ 1 & 2 & 8 & 0,345 & 0,652 & 0,003\end{array}$




\begin{tabular}{|c|c|c|c|c|c|}
\hline 2 & 4 & 11 & 0,455 & 0,495 & 0,05 \\
\hline 1 & 2 & 4 & 0,625 & 0,355 & 0,02 \\
\hline 2 & 12 & 16 & 0,845 & 0 & 0,155 \\
\hline 1 & 3 & 3 & 0,125 & 0,865 & 0,01 \\
\hline 1 & 5 & 12 & 0,024 & 0,452 & 0,524 \\
\hline 2 & 10 & 8 & 0,952 & 0 & 0,048 \\
\hline 2 & 1 & 5 & 0,952 & 0,015 & 0,033 \\
\hline 2 & 1 & 13 & 0,125 & 0,545 & 0,33 \\
\hline 2 & 5 & 2 & 0,952 & 0,015 & 0,033 \\
\hline 2 & 9 & 19 & 0,755 & 0,125 & 0,12 \\
\hline 2 & 6 & 5 & 0,75 & 0,015 & 0,235 \\
\hline 2 & 7 & 16 & 0,85 & 0 & 0,15 \\
\hline 2 & 2 & 11 & 0,125 & 0,855 & 0,02 \\
\hline 2 & 3 & 12 & 0,55 & 0,425 & 0,025 \\
\hline 2 & 8 & 6 & 0,625 & 0,125 & 0,25 \\
\hline 1 & 1 & 10 & 0,355 & 0,455 & 0,19 \\
\hline 2 & 2 & 9 & 0,525 & 0,425 & 0,05 \\
\hline 2 & 11 & 16 & 0,845 & 0 & 0,155 \\
\hline 2 & 1 & 17 & 0,755 & 0,125 & 0,12 \\
\hline 2 & 7 & 7 & 0,45 & 0,45 & 0,1 \\
\hline 2 & 8 & 1 & 0,655 & 0,245 & 0,1 \\
\hline 2 & 7 & 10 & 0,455 & 0,545 & $\begin{array}{l}-1,11 \mathrm{E}- \\
16\end{array}$ \\
\hline 1 & 5 & 20 & 0,856 & 0 & 0,144 \\
\hline 2 & 4 & 4 & 0,425 & 0,555 & 0,02 \\
\hline 2 & 5 & 15 & 0,955 & 0,015 & 0,03 \\
\hline 1 & 5 & 8 & 0,652 & 0,345 & 0,003 \\
\hline 2 & 5 & 13 & 0,125 & 0,545 & 0,33 \\
\hline 1 & 2 & 2 & 1 & 0 & 0 \\
\hline 1 & 3 & 10 & 0,455 & 0,455 & 0,09 \\
\hline 1 & 4 & 6 & 0,155 & 0,755 & 0,09 \\
\hline 2 & 6 & 4 & 0,555 & 0,425 & 0,02 \\
\hline 1 & 1 & 13 & 0,155 & 0,755 & 0,09 \\
\hline 2 & 10 & 12 & 0,865 & 0 & 0,135 \\
\hline 1 & 2 & 20 & 0,825 & 0 & 0,175 \\
\hline 1 & 3 & 6 & 0,055 & 0,755 & 0,19 \\
\hline 1 & 5 & 10 & 0,125 & 0,455 & 0,42 \\
\hline 2 & 2 & 1 & 0,855 & 0 & 0,145 \\
\hline 2 & 6 & 18 & 0,854 & 0,125 & 0,021 \\
\hline 2 & 8 & 18 & 0,125 & 0,855 & 0,02 \\
\hline 1 & 5 & 13 & 0,125 & 0,855 & 0,02 \\
\hline 1 & 6 & 9 & 0,125 & 0,855 & 0,02 \\
\hline 2 & 3 & 14 & 0,425 & 0,455 & 0,12 \\
\hline 2 & 9 & 15 & 0,015 & 0,955 & 0,03 \\
\hline
\end{tabular}




\begin{tabular}{|c|c|c|c|c|c|}
\hline 1 & 6 & 14 & 0,455 & 0,455 & 0,09 \\
\hline 1 & 1 & 4 & 0,255 & 0,625 & 0,12 \\
\hline 1 & 3 & 14 & 0,955 & 0 & 0,045 \\
\hline 2 & 5 & 9 & 0,325 & 0,525 & 0,15 \\
\hline 2 & 3 & 2 & 0,15 & 0,85 & 0 \\
\hline 2 & 4 & 5 & 0,853 & 0,015 & 0,132 \\
\hline 2 & 5 & 3 & 0,015 & 0,455 & 0,53 \\
\hline 2 & 5 & 5 & 0,952 & 0,015 & 0,033 \\
\hline 2 & 7 & 11 & 0,35 & 0,495 & 0,155 \\
\hline 1 & 6 & 8 & 0,736 & 0,245 & 0,019 \\
\hline 2 & 11 & 3 & 0,225 & 0,455 & 0,32 \\
\hline 1 & 1 & 16 & 0,925 & 0 & 0,075 \\
\hline 1 & 6 & 1 & 1 & 0 & 0 \\
\hline 1 & 6 & 11 & 0,721 & 0,145 & 0,134 \\
\hline 2 & 10 & 14 & 0,255 & 0,745 & 0 \\
\hline 1 & 3 & 9 & 0,855 & 0,125 & 0,02 \\
\hline 2 & 7 & 18 & 0,855 & 0,125 & 0,02 \\
\hline 1 & 3 & 16 & 0,254 & 0,652 & 0,094 \\
\hline 1 & 1 & 20 & 0,895 & 0 & 0,105 \\
\hline 1 & 6 & 19 & 0,785 & 0,125 & 0,09 \\
\hline 2 & 8 & 7 & 0,425 & 0,45 & 0,125 \\
\hline 2 & 10 & 17 & 0,95 & 0 & 0,05 \\
\hline 2 & 12 & 17 & 0,56 & 0,125 & 0,315 \\
\hline 1 & 4 & 20 & 0,645 & 0 & 0,355 \\
\hline 2 & 6 & 8 & 0,125 & 0,853 & 0,022 \\
\hline 2 & 9 & 13 & 0,425 & 0,545 & 0,03 \\
\hline 2 & 1 & 3 & 0,015 & 0,455 & 0,53 \\
\hline 2 & 8 & 13 & 0,125 & 0,545 & 0,33 \\
\hline 2 & 1 & 1 & 0,855 & 0 & 0,145 \\
\hline 2 & 4 & 14 & 0,255 & 0,745 & 0 \\
\hline 2 & 7 & 15 & 0,75 & 0,245 & 0,005 \\
\hline 2 & 10 & 15 & 0,855 & 0,125 & 0,02 \\
\hline 2 & 9 & 17 & 0,855 & 0,125 & 0,02 \\
\hline 2 & 1 & 7 & 0,425 & 0,45 & 0,125 \\
\hline 1 & 5 & 14 & 0,125 & 0,455 & 0,42 \\
\hline 1 & 6 & 6 & 0,355 & 0,355 & 0,29 \\
\hline 2 & 3 & 4 & 0,225 & 0,585 & 0,19 \\
\hline 2 & 5 & 6 & 0,625 & 0,125 & 0,25 \\
\hline 2 & 5 & 7 & 0,425 & 0,45 & 0,125 \\
\hline 2 & 12 & 13 & 0,545 & 0,425 & 0,03 \\
\hline 1 & 4 & 15 & 0,015 & 0,867 & 0,118 \\
\hline 1 & 6 & 10 & 0,255 & 0,455 & 0,29 \\
\hline 2 & 4 & 6 & 0,625 & 0,125 & 0,25 \\
\hline 2 & 5 & 1 & 0,245 & 0,655 & 0,1 \\
\hline
\end{tabular}




\begin{tabular}{|c|c|c|c|c|c|}
\hline 2 & 3 & 20 & 0,256 & 0,725 & 0,019 \\
\hline 2 & 4 & 13 & 0,125 & 0,545 & 0,33 \\
\hline 2 & 8 & 19 & 0,125 & 0,865 & 0,01 \\
\hline 2 & 2 & 12 & 0,55 & 0,425 & 0,025 \\
\hline 2 & 8 & 12 & 0,785 & 0 & 0,215 \\
\hline 2 & 7 & 2 & 0,85 & 0,15 & $\begin{array}{l}2,78 \mathrm{E}- \\
17\end{array}$ \\
\hline 2 & 12 & 18 & 0,325 & 0,55 & 0,125 \\
\hline 1 & 6 & 13 & 0,855 & 0,125 & 0,02 \\
\hline 2 & 1 & 11 & 0,125 & 0,855 & 0,02 \\
\hline 2 & 4 & 12 & 0,853 & 0 & 0,147 \\
\hline 2 & 8 & 14 & 0,345 & 0,455 & 0,2 \\
\hline 2 & 7 & 19 & 0,985 & 0 & 0,015 \\
\hline 1 & 1 & 19 & 0,91 & 0 & 0,09 \\
\hline 1 & 3 & 19 & 0,254 & 0,569 & 0,177 \\
\hline 2 & 2 & 6 & 0,015 & 0,625 & 0,36 \\
\hline 2 & 2 & 18 & 0,55 & 0,325 & 0,125 \\
\hline 2 & 12 & 9 & 0,825 & 0 & 0,175 \\
\hline 1 & 1 & 14 & 0,955 & 0 & 0,045 \\
\hline 2 & 1 & 15 & 0,855 & 0,125 & 0,02 \\
\hline 1 & 4 & 17 & 0,254 & 0,698 & 0,048 \\
\hline 2 & 7 & 8 & 0,785 & 0,15 & 0,065 \\
\hline 2 & 5 & 18 & 0,745 & 0,255 & 0 \\
\hline 2 & 9 & 3 & 0,455 & 0,455 & 0,09 \\
\hline 2 & 12 & 19 & 0,125 & 0,865 & 0,01 \\
\hline 1 & 4 & 4 & 0,855 & 0 & 0,145 \\
\hline 1 & 5 & 2 & 0 & 1 & 0 \\
\hline 1 & 1 & 6 & 0,755 & 0,155 & 0,09 \\
\hline 1 & 3 & 4 & 0,355 & 0,625 & 0,02 \\
\hline 2 & 2 & 19 & 0,865 & 0,125 & 0,01 \\
\hline 2 & 5 & 11 & 0,495 & 0,495 & 0,01 \\
\hline 2 & 6 & 2 & 0,895 & 0,015 & 0,09 \\
\hline 2 & 1 & 16 & 0,425 & 0,425 & 0,15 \\
\hline 2 & 4 & 10 & 0,825 & 0,15 & 0,025 \\
\hline 1 & 4 & 14 & 0,455 & 0,455 & 0,09 \\
\hline 1 & 6 & 2 & 0 & 1 & 0 \\
\hline 1 & 2 & 18 & 0,021 & 0,654 & 0,325 \\
\hline 1 & 5 & 6 & 0,255 & 0,355 & 0,39 \\
\hline 1 & 6 & 18 & 0,995 & 0,001 & 0,004 \\
\hline 2 & 3 & 19 & 0,985 & 0 & 0,015 \\
\hline 2 & 12 & 8 & 0,853 & 0,125 & 0,022 \\
\hline 2 & 11 & 6 & 0,625 & 0,125 & 0,25 \\
\hline 2 & 7 & 3 & 0,225 & 0,455 & 0,32 \\
\hline 1 & 1 & 18 & 0,001 & 0,995 & 0,004 \\
\hline
\end{tabular}




\begin{tabular}{|c|c|c|c|c|c|}
\hline 2 & 12 & 5 & 0,845 & 0,015 & 0,14 \\
\hline 1 & 5 & 16 & 0,256 & 0,652 & 0,092 \\
\hline 1 & 1 & 7 & 0,625 & 0,255 & 0,12 \\
\hline 2 & 10 & 3 & 0,225 & 0,455 & 0,32 \\
\hline 2 & 12 & 1 & 0,852 & 0 & 0,148 \\
\hline 2 & 8 & 9 & 0,845 & 0 & 0,155 \\
\hline 1 & 5 & 1 & 1 & 0 & 0 \\
\hline 1 & 5 & 9 & 0,008 & 0,855 & 0,137 \\
\hline 2 & 2 & 14 & 0,425 & 0,455 & 0,12 \\
\hline 2 & 3 & 7 & 0,225 & 0,45 & 0,325 \\
\hline 2 & 5 & 8 & 0,125 & 0,853 & 0,022 \\
\hline 2 & 9 & 14 & 0,125 & 0,745 & 0,13 \\
\hline 2 & 8 & 10 & 0,545 & 0,455 & $\begin{array}{l}-5,55 \mathrm{E}- \\
17\end{array}$ \\
\hline 2 & 11 & 9 & 0,325 & 0,525 & 0,15 \\
\hline 2 & 7 & 5 & 0,85 & 0,015 & 0,135 \\
\hline 1 & 2 & 6 & 0,125 & 0,755 & 0,12 \\
\hline 2 & 2 & 20 & 0,325 & 0,546 & 0,129 \\
\hline 2 & 4 & 9 & 0,325 & 0,525 & 0,15 \\
\hline 2 & 9 & 20 & 0,125 & 0,725 & 0,15 \\
\hline 1 & 4 & 11 & 0,865 & 0 & 0,135 \\
\hline 2 & 6 & 1 & 0,855 & 0 & 0,145 \\
\hline 2 & 2 & 17 & 0,75 & 0,125 & 0,125 \\
\hline 2 & 9 & 11 & 0,15 & 0,495 & 0,355 \\
\hline 2 & 11 & 18 & 0,125 & 0,855 & 0,02 \\
\hline 2 & 9 & 6 & 0,525 & 0,125 & 0,35 \\
\hline 2 & 5 & 14 & 0,255 & 0,745 & 0 \\
\hline 2 & 9 & 5 & 0,015 & 0,952 & 0,033 \\
\hline 1 & 6 & 3 & 0,005 & 0,865 & 0,13 \\
\hline 2 & 1 & 8 & 0,853 & 0,125 & 0,022 \\
\hline 2 & 4 & 1 & 0,555 & 0,425 & 0,02 \\
\hline 1 & 1 & 5 & 0,985 & 0 & 0,015 \\
\hline 2 & 8 & 3 & 0,245 & 0,455 & 0,3 \\
\hline 2 & 11 & 1 & 0,425 & 0,555 & 0,02 \\
\hline 1 & 2 & 9 & 0,125 & 0,855 & 0,02 \\
\hline 2 & 12 & 12 & 0,845 & 0 & 0,155 \\
\hline 2 & 12 & 20 & 0,125 & 0,725 & 0,15 \\
\hline 1 & 2 & 11 & 0,852 & 0,145 & 0,003 \\
\hline 2 & 10 & 9 & 0,425 & 0,525 & 0,05 \\
\hline 1 & 5 & 19 & 0,854 & 0,125 & 0,021 \\
\hline 1 & 3 & 1 & 1 & 0 & 0 \\
\hline 1 & 5 & 5 & 0,255 & 0,455 & 0,29 \\
\hline 2 & 2 & 15 & 0,753 & 0,245 & 0,002 \\
\hline 2 & 3 & 6 & 0,015 & 0,625 & 0,36 \\
\hline
\end{tabular}




\begin{tabular}{|c|c|c|c|c|c|}
\hline 2 & 5 & 12 & 0,952 & 0 & 0,048 \\
\hline 2 & 2 & 3 & 0,015 & 0,455 & 0,53 \\
\hline 2 & 6 & 10 & 0,825 & 0,15 & 0,025 \\
\hline 2 & 11 & 7 & 0,425 & 0,45 & 0,125 \\
\hline 2 & 3 & 13 & 0,125 & 0,545 & 0,33 \\
\hline 1 & 4 & 16 & 0,845 & 0 & 0,155 \\
\hline 2 & 10 & 5 & 0,865 & 0,015 & 0,12 \\
\hline 2 & 12 & 10 & 0,425 & 0,545 & 0,03 \\
\hline 2 & 8 & 17 & 0,125 & 0,855 & 0,02 \\
\hline 2 & 11 & 19 & 0,825 & 0,125 & 0,05 \\
\hline 2 & 11 & 13 & 0,125 & 0,545 & 0,33 \\
\hline 1 & 3 & 5 & 0,854 & 0 & 0,146 \\
\hline 2 & 3 & 16 & 0,75 & 0 & 0,25 \\
\hline 2 & 5 & 19 & 0,845 & 0,125 & 0,03 \\
\hline 2 & 11 & 11 & 0,255 & 0,495 & 0,25 \\
\hline 2 & 12 & 11 & 0,355 & 0,495 & 0,15 \\
\hline 2 & 2 & 4 & 0,225 & 0,585 & 0,19 \\
\hline 2 & 7 & 4 & 0,425 & 0,555 & 0,02 \\
\hline 2 & 6 & 6 & 0,625 & 0,125 & 0,25 \\
\hline 2 & 7 & 17 & 0,985 & 0 & 0,015 \\
\hline 1 & 4 & 2 & 0 & 1 & 0 \\
\hline 2 & 3 & 5 & 0,952 & 0,015 & 0,033 \\
\hline 2 & 7 & 14 & 0,455 & 0,455 & 0,09 \\
\hline 1 & 1 & 2 & 1 & 0 & 0 \\
\hline 1 & 3 & 12 & 0,256 & 0,452 & 0,292 \\
\hline 2 & 3 & 3 & 0,015 & 0,455 & 0,53 \\
\hline 2 & 11 & 14 & 0,255 & 0,745 & 0 \\
\hline 2 & 1 & 19 & 0,855 & 0,125 & 0,02 \\
\hline 1 & 3 & 13 & 0,325 & 0,325 & 0,35 \\
\hline 2 & 1 & 6 & 0,125 & 0,625 & 0,25 \\
\hline 2 & 1 & 20 & 0,546 & 0,325 & 0,129 \\
\hline 1 & 2 & 12 & 0,125 & 0,452 & 0,423 \\
\hline 2 & 6 & 14 & 0,745 & 0,255 & 0 \\
\hline 1 & 1 & 12 & 0,025 & 0,452 & 0,523 \\
\hline 2 & 11 & 4 & 0,425 & 0,555 & 0,02 \\
\hline 2 & 10 & 6 & 0,015 & 0,625 & 0,36 \\
\hline 1 & 4 & 10 & 0,255 & 0,455 & 0,29 \\
\hline 2 & 12 & 14 & 0,345 & 0,455 & 0,2 \\
\hline 1 & 1 & 8 & 0,245 & 0,736 & 0,019 \\
\hline 2 & 11 & 12 & 0,845 & 0 & 0,155 \\
\hline 2 & 6 & 13 & 0,125 & 0,545 & 0,33 \\
\hline 2 & 8 & 8 & 0,125 & 0,853 & 0,022 \\
\hline 2 & 10 & 16 & 0,865 & 0 & 0,135 \\
\hline 2 & 3 & 1 & 0,925 & 0 & 0,075 \\
\hline
\end{tabular}




\begin{tabular}{|c|c|c|c|c|c|}
\hline 2 & 3 & 9 & 0,525 & 0,425 & 0,05 \\
\hline 1 & 2 & 16 & 0,652 & 0,256 & 0,092 \\
\hline 2 & 2 & 7 & 0,225 & 0,45 & 0,325 \\
\hline 1 & 3 & 20 & 0,785 & 0 & 0,215 \\
\hline 2 & 4 & 18 & 0,855 & 0,125 & 0,02 \\
\hline 1 & 4 & 19 & 0,125 & 0,854 & 0,021 \\
\hline 2 & 4 & 8 & 0,125 & 0,853 & 0,022 \\
\hline 2 & 12 & 7 & 0,425 & 0,45 & 0,125 \\
\hline 2 & 1 & 14 & 0,255 & 0,745 & 0 \\
\hline 1 & 4 & 1 & 1 & 0 & 0 \\
\hline 1 & 4 & 5 & 0,245 & 0,455 & 0,3 \\
\hline 2 & 7 & 6 & 0,545 & 0,125 & 0,33 \\
\hline 2 & 8 & 20 & 0,125 & 0,725 & 0,15 \\
\hline 1 & 1 & 3 & 0,001 & 0,865 & 0,134 \\
\hline 2 & 10 & 2 & 0,865 & 0,015 & 0,12 \\
\hline 2 & 6 & 3 & 0,325 & 0,455 & 0,22 \\
\hline 2 & 6 & 16 & 0,895 & 0 & 0,105 \\
\hline 2 & 10 & 7 & 0,225 & 0,45 & 0,325 \\
\hline 2 & 10 & 18 & 0,125 & 0,855 & 0,02 \\
\hline 1 & 4 & 13 & 0,855 & 0,125 & 0,02 \\
\hline 1 & 5 & 18 & 0,985 & 0,001 & 0,014 \\
\hline 2 & 3 & 8 & 0,952 & 0 & 0,048 \\
\hline 2 & 10 & 1 & 0,525 & 0,425 & 0,05 \\
\hline 2 & 9 & 18 & 0,255 & 0,745 & 0 \\
\hline 2 & 3 & 10 & 0,325 & 0,545 & 0,13 \\
\hline 2 & 4 & 19 & 0,845 & 0,125 & 0,03 \\
\hline 2 & 8 & 11 & 0,45 & 0,495 & 0,055 \\
\hline 1 & 3 & 8 & 0,945 & 0 & 0,055 \\
\hline 1 & 6 & 17 & 0,845 & 0 & 0,155 \\
\hline 2 & 5 & 10 & 0,825 & 0,15 & 0,025 \\
\hline 2 & 8 & 5 & 0,785 & 0,015 & 0,2 \\
\hline 1 & 6 & 7 & 0,5 & 0,5 & 0 \\
\hline 2 & 1 & 9 & 0,745 & 0 & 0,255 \\
\hline 1 & 4 & 3 & 0,125 & 0,865 & 0,01 \\
\hline 2 & 9 & 9 & 0,425 & 0,525 & 0,05 \\
\hline 1 & 2 & 3 & 0,865 & 0,125 & 0,01 \\
\hline 2 & 1 & 10 & 0,525 & 0,455 & 0,02 \\
\hline 1 & 1 & 15 & 0,125 & 0,867 & 0,008 \\
\hline 2 & 5 & 17 & 0,745 & 0,125 & 0,13 \\
\hline 2 & 7 & 1 & 0,955 & 0 & 0,045 \\
\hline 1 & 1 & 11 & 0,785 & 0,145 & 0,07 \\
\hline 1 & 5 & 3 & 0,015 & 0,865 & 0,12 \\
\hline 2 & 5 & 4 & 0,225 & 0,585 & 0,19 \\
\hline 2 & 9 & 7 & 0,125 & 0,45 & 0,425 \\
\hline
\end{tabular}




\begin{tabular}{|c|c|c|c|c|c|}
\hline 1 & 4 & 7 & 0,455 & 0,5 & 0,045 \\
\hline 2 & 1 & 2 & 0,245 & 0,245 & 0,51 \\
\hline 1 & 4 & 12 & 0,452 & 0,452 & 0,096 \\
\hline 1 & 5 & 11 & 0,912 & 0 & 0,088 \\
\hline 1 & 6 & 20 & 0,886 & 0 & 0,114 \\
\hline 2 & 10 & 11 & 0,015 & 0,855 & 0,13 \\
\hline 2 & 12 & 4 & 0,425 & 0,555 & 0,02 \\
\hline 2 & 5 & 20 & 0,325 & 0,546 & 0,129 \\
\hline 1 & 2 & 13 & 0,755 & 0,155 & 0,09 \\
\hline 2 & 11 & 15 & 0,85 & 0,125 & 0,025 \\
\hline 2 & 7 & 13 & 0,225 & 0,545 & 0,23 \\
\hline 2 & 4 & 15 & 0,952 & 0,015 & 0,033 \\
\hline 2 & 10 & 13 & 0,125 & 0,545 & 0,33 \\
\hline 1 & 3 & 15 & 0,255 & 0,352 & 0,393 \\
\hline 2 & 3 & 11 & 0,125 & 0,855 & 0,02 \\
\hline 2 & 8 & 16 & 0,785 & 0 & 0,215 \\
\hline 1 & 6 & 12 & 0,362 & 0,452 & 0,186 \\
\hline 2 & 11 & 17 & 0,725 & 0,125 & 0,15 \\
\hline 1 & 3 & 17 & 0,985 & 0 & 0,015 \\
\hline 2 & 4 & 3 & 0,125 & 0,455 & 0,42 \\
\hline 2 & 4 & 7 & 0,425 & 0,45 & 0,125 \\
\hline 2 & 7 & 20 & 0,725 & 0,256 & 0,019 \\
\hline 2 & 10 & 10 & 0,15 & 0,845 & 0,005 \\
\hline 1 & 6 & 16 & 0,352 & 0,352 & 0,296 \\
\hline 2 & 8 & 2 & 0,785 & 0,15 & 0,065 \\
\hline 1 & 2 & 17 & 0,698 & 0,254 & 0,048 \\
\hline 1 & 2 & 19 & 0,569 & 0,254 & 0,177 \\
\hline 2 & 2 & 16 & 0,75 & 0 & 0,25 \\
\hline 2 & 2 & 5 & 0,952 & 0,015 & 0,033 \\
\hline 2 & 2 & 13 & 0,125 & 0,545 & 0,33 \\
\hline 2 & 7 & 12 & 0,85 & 0 & 0,15 \\
\hline 2 & 1 & 18 & 0,75 & 0,125 & 0,125 \\
\hline 1 & 2 & 1 & 0 & 1 & 0 \\
\hline 1 & 4 & 8 & 0,845 & 0 & 0,155 \\
\hline 2 & 1 & 4 & 0,225 & 0,585 & 0,19 \\
\hline 2 & 4 & 16 & 0,853 & 0 & 0,147 \\
\hline 1 & 5 & 7 & 0,455 & 0,5 & 0,045 \\
\hline 2 & 6 & 19 & 0,752 & 0,125 & 0,123 \\
\hline 2 & 10 & 4 & 0,425 & 0,555 & 0,02 \\
\hline 1 & 6 & 5 & 0,455 & 0,455 & 0,09 \\
\hline 1 & 1 & 9 & 0,255 & 0,255 & 0,49 \\
\hline 2 & 4 & 2 & 0,853 & 0,015 & 0,132 \\
\hline 2 & 9 & 12 & 0,425 & 0,55 & 0,025 \\
\hline 2 & 9 & 10 & 0,125 & 0,845 & 0,03 \\
\hline
\end{tabular}




\begin{tabular}{|c|c|c|c|c|c|}
\hline 2 & 9 & 16 & 0,925 & 0 & 0,075 \\
\hline 1 & 2 & 7 & 0,945 & 0 & 0,055 \\
\hline 2 & 7 & 9 & 0,825 & 0 & 0,175 \\
\hline 1 & 2 & 14 & 0,455 & 0,455 & 0,09 \\
\hline 2 & 4 & 17 & 0,745 & 0,125 & 0,13 \\
\hline 2 & 2 & 2 & 0,015 & 0,952 & 0,033 \\
\hline 2 & 11 & 10 & 0,845 & 0,15 & 0,005 \\
\hline 2 & 6 & 15 & 0,125 & 0,855 & 0,02 \\
\hline 2 & 6 & 9 & 0,325 & 0,525 & 0,15 \\
\hline 2 & 11 & 8 & 0,125 & 0,853 & 0,022 \\
\hline 2 & 3 & 18 & 0,455 & 0,455 & 0,09 \\
\hline 2 & 6 & 11 & 0,855 & 0,125 & 0,02 \\
\hline 2 & 12 & 6 & 0,125 & 0,625 & 0,25 \\
\hline 2 & 3 & 15 & 0,753 & 0,245 & 0,002 \\
\hline 2 & 8 & 15 & 0,85 & 0,125 & 0,025 \\
\hline 2 & 1 & 12 & 0,35 & 0,55 & 0,1 \\
\hline 1 & 5 & 17 & 0,456 & 0,456 & 0,088 \\
\hline 1 & 3 & 11 & 0,145 & 0,852 & 0,003 \\
\hline 1 & 5 & 4 & 0,985 & 0 & 0,015 \\
\hline 2 & 9 & 1 & 0,15 & 0,655 & 0,195 \\
\hline 1 & 4 & 18 & 0,654 & 0,021 & 0,325 \\
\hline 1 & 3 & 2 & 0 & 1 & 0 \\
\hline 2 & 6 & 12 & 0,895 & 0 & 0,105 \\
\hline 2 & 2 & 10 & 0,325 & 0,545 & 0,13 \\
\hline 2 & 6 & 17 & 0,85 & 0,125 & 0,025 \\
\hline 2 & 11 & 20 & 0 & 0,725 & 0,275 \\
\hline 2 & 12 & 3 & 0,225 & 0,455 & 0,32 \\
\hline 2 & 2 & 8 & 0,952 & 0 & 0,048 \\
\hline 2 & 6 & 7 & 0,425 & $\begin{array}{c}0,45 \\
\text { Table-7 }\end{array}$ & 0,125 \\
\hline
\end{tabular}

The clusters obtained as in Table-7 are intuitive fuzzy sets.

Description-3

When Hamming measure is applied to these clusters using definition-3, the relationship between A and $S$ can be seen as in Table-8.

$\begin{array}{lllll}\text { DRINK_ID } & \text { DRINK } & \text { FOOD_ID } & \text { FOOD } & \text { DISTANCE } \\ \mathbf{4} & \begin{array}{l}\text { DRINK } \\ \text { NO.4 }\end{array} & 4 & \text { CHICKEN } & 21,062 \\ \mathbf{2} & \begin{array}{l}\text { DRINK } \\ \text { NO.2 }\end{array} & 4 & \text { CHICKEN } & 18,612 \\ \mathbf{3} & \begin{array}{l}\text { DRINK } \\ \text { DO.3 }\end{array} & 4 & \text { CHICKEN } & 18,516 \\ \mathbf{5} & \text { NRINK } & 4 & \text { CHICKEN } & 17,674 \\ \mathbf{6} & \text { NO.5 } & & & \\ & \text { DRINK } & 4 & \text { CHICKEN } & 17,41\end{array}$




\begin{tabular}{|c|c|c|c|c|}
\hline & NO.6 & & & \\
\hline 1 & $\begin{array}{l}\text { DRINK } \\
\text { NO.1 }\end{array}$ & 4 & CHICKEN & 15,002 \\
\hline 2 & $\begin{array}{l}\text { DRINK } \\
\text { NO.2 }\end{array}$ & 7 & CHEESE & 19,302 \\
\hline 4 & $\begin{array}{l}\text { DRINK } \\
\text { NO.4 }\end{array}$ & 7 & CHEESE & 18,028 \\
\hline 5 & $\begin{array}{l}\text { DRINK } \\
\text { NO.5 }\end{array}$ & 7 & CHEESE & 16,242 \\
\hline 1 & $\begin{array}{l}\text { DRINK } \\
\text { NO.1 }\end{array}$ & 7 & CHEESE & 15,434 \\
\hline 6 & $\begin{array}{l}\text { DRINK } \\
\text { N0.6 }\end{array}$ & 7 & CHEESE & 14,214 \\
\hline 3 & $\begin{array}{l}\text { DRINK } \\
\text { NO.3 }\end{array}$ & 7 & CHEESE & 13,11 \\
\hline 5 & $\begin{array}{l}\text { DRINK } \\
\text { NO.5 }\end{array}$ & 6 & FISH & 18,3 \\
\hline 4 & $\begin{array}{l}\text { DRINK } \\
\text { NO.4 }\end{array}$ & 6 & FISH & 18,088 \\
\hline 2 & $\begin{array}{l}\text { DRINK } \\
\text { NO.2 }\end{array}$ & 6 & FISH & 17,864 \\
\hline 3 & $\begin{array}{l}\text { DRINK } \\
\text { NO.3 }\end{array}$ & 6 & FISH & 17,774 \\
\hline 6 & $\begin{array}{l}\text { DRINK } \\
\text { N0.6 }\end{array}$ & 6 & FISH & 16,218 \\
\hline 1 & $\begin{array}{l}\text { DRINK } \\
\text { NO.1 }\end{array}$ & 6 & FISH & 13,61 \\
\hline 6 & $\begin{array}{l}\text { DRINK } \\
\text { N0.6 }\end{array}$ & 8 & FRUIT & 20,962 \\
\hline 5 & $\begin{array}{l}\text { DRINK } \\
\text { NO.5 }\end{array}$ & 8 & FRUIT & 19,944 \\
\hline 2 & $\begin{array}{l}\text { DRINK } \\
\text { NO.2 }\end{array}$ & 8 & FRUIT & 19,186 \\
\hline 4 & $\begin{array}{l}\text { DRINK } \\
\text { NO.4 }\end{array}$ & 8 & FRUIT & 19,174 \\
\hline 3 & $\begin{array}{l}\text { DRINK } \\
\text { NO.3 }\end{array}$ & 8 & FRUIT & 19,122 \\
\hline 1 & $\begin{array}{l}\text { DRINK } \\
\text { NO.1 }\end{array}$ & 8 & FRUIT & 17,284 \\
\hline 2 & $\begin{array}{l}\text { DRINK } \\
\text { NO.2 }\end{array}$ & 3 & VENISON & 20,774 \\
\hline 1 & $\begin{array}{l}\text { DRINK } \\
\text { NO.1 }\end{array}$ & 3 & VENISON & 18,044 \\
\hline 4 & $\begin{array}{l}\text { DRINK } \\
\text { NO.4 }\end{array}$ & 3 & VENISON & 17,184 \\
\hline 5 & $\begin{array}{l}\text { DRINK } \\
\text { NO.5 }\end{array}$ & 3 & VENISON & 16,798 \\
\hline 6 & $\begin{array}{l}\text { DRINK } \\
\text { NO.6 }\end{array}$ & 3 & VENISON & 14,89 \\
\hline
\end{tabular}




\begin{tabular}{|c|c|c|c|c|}
\hline 3 & $\begin{array}{l}\text { DRINK } \\
\text { NO.3 }\end{array}$ & 3 & VENISON & 12,33 \\
\hline 2 & $\begin{array}{l}\text { DRINK } \\
\text { NO.2 }\end{array}$ & 2 & $\begin{array}{l}\text { OILY RED } \\
\text { MEAT }\end{array}$ & 20,386 \\
\hline 1 & $\begin{array}{l}\text { DRINK } \\
\text { NO.1 }\end{array}$ & 2 & $\begin{array}{l}\text { OILY RED } \\
\text { MEAT }\end{array}$ & 18,404 \\
\hline 4 & $\begin{array}{l}\text { DRINK } \\
\text { NO.4 }\end{array}$ & 2 & $\begin{array}{l}\text { OILY RED } \\
\text { MEAT }\end{array}$ & 16,012 \\
\hline 5 & $\begin{array}{l}\text { DRINK } \\
\text { NO.5 }\end{array}$ & 2 & $\begin{array}{l}\text { OILY RED } \\
\text { MEAT }\end{array}$ & 15,696 \\
\hline 6 & $\begin{array}{l}\text { DRINK } \\
\text { NO.6 }\end{array}$ & 2 & $\begin{array}{l}\text { OILY RED } \\
\text { MEAT }\end{array}$ & 14,228 \\
\hline 3 & $\begin{array}{l}\text { DRINK } \\
\text { NO.3 }\end{array}$ & 2 & $\begin{array}{l}\text { OILY RED } \\
\text { MEAT }\end{array}$ & 11,758 \\
\hline 4 & $\begin{array}{l}\text { DRINK } \\
\text { NO.4 }\end{array}$ & 10 & PORK & 20,64 \\
\hline 5 & $\begin{array}{l}\text { DRINK } \\
\text { NO.5 }\end{array}$ & 10 & PORK & 19,416 \\
\hline 6 & $\begin{array}{l}\text { DRINK } \\
\text { N0.6 }\end{array}$ & 10 & PORK & 18,72 \\
\hline 2 & $\begin{array}{l}\text { DRINK } \\
\text { NO.2 }\end{array}$ & 10 & PORK & 18,206 \\
\hline 1 & $\begin{array}{l}\text { DRINK } \\
\text { NO.1 }\end{array}$ & 10 & PORK & 16,454 \\
\hline 3 & $\begin{array}{l}\text { DRINK } \\
\text { NO.3 }\end{array}$ & 10 & PORK & 16,08 \\
\hline 2 & $\begin{array}{l}\text { DRINK } \\
\text { NO.2 }\end{array}$ & 1 & RED MEAT & 20,526 \\
\hline 4 & $\begin{array}{l}\text { DRINK } \\
\text { NO.4 }\end{array}$ & 1 & RED MEAT & 18,65 \\
\hline 1 & $\begin{array}{l}\text { DRINK } \\
\text { NO.1 }\end{array}$ & 1 & RED MEAT & 18,396 \\
\hline 5 & $\begin{array}{l}\text { DRINK } \\
\text { NO.5 }\end{array}$ & 1 & RED MEAT & 15,464 \\
\hline 6 & $\begin{array}{l}\text { DRINK } \\
\text { N0.6 }\end{array}$ & 1 & RED MEAT & 15,1 \\
\hline 3 & $\begin{array}{l}\text { DRINK } \\
\text { NO.3 }\end{array}$ & 1 & RED MEAT & 11,666 \\
\hline 4 & $\begin{array}{l}\text { DRINK } \\
\text { NO.4 }\end{array}$ & 11 & SHRIMP & 22,502 \\
\hline 3 & $\begin{array}{l}\text { DRINK } \\
\text { NO.3 }\end{array}$ & 11 & SHRIMP & 20,264 \\
\hline 5 & $\begin{array}{l}\text { DRINK } \\
\text { NO.5 }\end{array}$ & 11 & SHRIMP & 19,93 \\
\hline 6 & $\begin{array}{l}\text { DRINK } \\
\text { N0.6 }\end{array}$ & 11 & SHRIMP & 19,506 \\
\hline 2 & $\begin{array}{l}\text { DRINK } \\
\text { NO.2 }\end{array}$ & 11 & SHRIMP & 17,832 \\
\hline 1 & DRINK & 11 & SHRIMP & 14,144 \\
\hline
\end{tabular}




\begin{tabular}{|c|c|c|c|c|}
\hline & N0.1 & & & \\
\hline 5 & $\begin{array}{l}\text { DRINK } \\
\text { NO.5 }\end{array}$ & 12 & SPICE & 20,286 \\
\hline 2 & $\begin{array}{l}\text { DRINK } \\
\text { NO.2 }\end{array}$ & 12 & SPICE & 18,256 \\
\hline 1 & $\begin{array}{l}\text { DRINK } \\
\text { NO.1 }\end{array}$ & 12 & SPICE & 17,602 \\
\hline 6 & $\begin{array}{l}\text { DRINK } \\
\text { NO.6 }\end{array}$ & 12 & SPICE & 17,322 \\
\hline 4 & $\begin{array}{l}\text { DRINK } \\
\text { NO.4 }\end{array}$ & 12 & SPICE & 15,002 \\
\hline 3 & $\begin{array}{l}\text { DRINK } \\
\text { NO.3 }\end{array}$ & 12 & SPICE & 14,406 \\
\hline 4 & $\begin{array}{l}\text { DRINK } \\
\text { NO.4 }\end{array}$ & 5 & TURKEY & 22,59 \\
\hline 3 & $\begin{array}{l}\text { DRINK } \\
\text { NO.3 }\end{array}$ & 5 & TURKEY & 20,296 \\
\hline 5 & $\begin{array}{l}\text { DRINK } \\
\text { NO.5 }\end{array}$ & 5 & TURKEY & 19,426 \\
\hline 6 & $\begin{array}{l}\text { DRINK } \\
\text { NO.6 }\end{array}$ & 5 & TURKEY & 19,124 \\
\hline 2 & $\begin{array}{l}\text { DRINK } \\
\text { NO.2 }\end{array}$ & 5 & TURKEY & 18,764 \\
\hline 1 & $\begin{array}{l}\text { DRINK } \\
\text { NO.1 }\end{array}$ & 5 & TURKEY & 13,802 \\
\hline 3 & $\begin{array}{l}\text { DRINK } \\
\text { NO.3 }\end{array}$ & 9 & VEGETABLES & 21,66 \\
\hline 5 & $\begin{array}{l}\text { DRINK } \\
\text { NO.5 }\end{array}$ & 9 & VEGETABLES & 21,222 \\
\hline 4 & $\begin{array}{l}\text { DRINK } \\
\text { NO.4 }\end{array}$ & 9 & VEGETABLES & 20,454 \\
\hline 6 & $\begin{array}{l}\text { DRINK } \\
\text { NO.6 }\end{array}$ & 9 & VEGETABLES & 19,152 \\
\hline 2 & $\begin{array}{l}\text { DRINK } \\
\text { NO.2 }\end{array}$ & 9 & VEGETABLES & 16,362 \\
\hline 1 & $\begin{array}{l}\text { DRINK } \\
\text { NO.1 }\end{array}$ & 9 & VEGETABLES & 14,832 \\
\hline
\end{tabular}

Table-8

When the results in Table- 8 are evaluated, the beverage consumed with "Chicken" food should be Drink no.4. It is clear that Drink no.1 and Drink no.2 drinks can be found in a service with "oily red meat" and "Venison", considering that there will be no fish and red meat in the same service, but fish and vegetable dishes.

As a different evaluation of the results, considering the Table-8, it can be considered that the Drink no. 1 drink and the Drink no. 2 drink have similar properties. 


\section{CONCLUSION}

It is considered as an important problem that data with different characteristics reach the same results in studies conducted through the Hamming measure. In this study, it will be more difficult to associate data with different characteristics with the same data, since the data that does not provide a characteristic by means of controlled sets is expressed with data within its own universal region. At the very least, it is not possible for elements that control each other to match the same data. The method followed in this study can be tested by using it in old applications. In addition, these criteria can be taken into account so that they can be easily applied to multi-criteria decision making problems in future studies.

\section{ACKNOWLEDGMENTS}

The authors would like to thank the reviewers and editors of Journal of Universal Mathematics.

\section{Funding}

This research was funded by Minevino co-Moldova, under grant no. MNV-2021-1001.

\section{The Declaration of Conflict of Interest Common Interest}

The author(s) declared that no conflict of interest or common interest.

\section{The Declaration of Ethics Committee Approval}

This study does not be necessary ethical committee permission or any special permission.

\section{The Declaration of Research and Publication Ethics}

The author(s) declared that they comply with the scientific, ethical, and citation rules of Journal of Universal Mathematics in all processes of the study and that they do not make any falsification on the data collected. Besides, the author(s) declared that Journal of Universal Mathematics and its editorial board have no responsibility for any ethical violations that may be encountered and this study has not been evaluated in any academic publication environment other than Journal of Universal Mathematics.

\section{REFERENCES}

[1] K. Atanassov, On Intuitionistic fuzzy sets theory, Springer, Berlin, (2012).

[2] K. Atanassov, Intuitionistic fuzzy logics as tools for evaluation of Data Mining processes, Knowledge-Based Systems, Vol. 80, pp.122-130, (2015).

[3] G. Çuvalcıoğlu, Some properties of Controlled Set Theory, Notes on Intuitionistic Fuzzy Sets ISSN 13104926 Vol. 20, No. 2, 37-42, (2014).

[4] P.A. Ejegwa, P. A., I.C. Onyeke, An Object Oriented Approach to the Application of Intuitionistic Fuzzy Sets in Competency Based Test Evaluation, Annals of Communications in Mathematics Volume 1, Number 1, 38-47, (2018).

[5] D. Li, C. Cheng, New similarity measures of intuitionistic fuzzy sets and application to pattern recognitions, Pattern Recog. Lett., 23, 221-225, (2002). 


\section{DECISION MAKING PROCESS VIA CONTROLLED SETS}

${ }^{1}$ DEPARTMENT OF MATHEMATICS, MERSIN UNIVERSITY, MERSIN,33016, TURKEY

Email address: gcuvalcioglu@mersin.edu.tr

2 MAJOR TEAM CO.(CEO), İSTANBUL,34061, TURKEY

Email address: ural@ minevino.com 